\title{
1 Einführung in und Überblick über das naturphilosophische Hauptwerk Die Stufen des Organischen und der Mensch
}

\subsection{Zum Thema des Lebens}

Das Leben ist heute nicht mehr wie im ersten Drittel des 20. Jahrhunderts ein Zauberwort, in dessen Zeichen man sich eine quasi mythologische Einheit gegen die dualistischen Trennungen in Materie und Geist, in Physisches und Psychisches versprechen konnte. Das Thema des Lebens war schon damals Gegenstand von vergleichsweise begrenzten Rationalisierungsprozessen und wurde in seiner unmittelbaren Mobilisierung sogleich aufgeteilt in arten-, rassen-, klassen- und kulturantagonistische Auslegungen. Der Kampf der je eigenen Interpretation gegen andere ideologische Deutungen des Lebens ging in Kriegen und im Zivilisationsbruch unter. Am Ende des 20. Jahrhunderts schien es so, als könne das Leben mehr und mehr zu fließen und zu gedeihen kanalisiert werden. Die neoliberale Globalisierung versprach allgemeine Prosperität im Leben früher oder später überall. Die biologische Rationalisierung des Lebens wurde unter dem medizinischen Vorzeichen zu helfen akzeptabel gemacht. Die Demokratie schien, sich von selbst auszubreiten, und die nachwachsenden Generationen begeisterten sich an der Utopie, in der digitalen Community der Transparenz wie in einer Schwarmintelligenz aufgehen zu dürfen.

Nachdem im letzten Jahrzehnt diese Versprechen einer allgemeinen Verbesserung des Lebens in die Permanenz von Krisenmodi geführt haben, liegt die Frage erneut nahe, was es mit dem Thema des Lebens in seinen vielfältigen Dimensionen grundsätzlich auf sich hat. Leben als Objekt zu rationalisieren und - an es appellierend - es als Subjekt zu mobilisieren, beides hat Grenzen, die einzusehen einen anderen philosophischen Weg als den dualistisch trennenden oder den einheitsmythologischen Zugang erfordert. Die Grundüberzeugung von Helmuth Plessner bestand darin, Lebensphänomene gerade dann qualitativ erfahren, differenziert verstehen und in ihren kritischen Veränderungen begreifen zu können, wenn sie weder in dualistische Trennungen noch in Einheitsmythen aufgelöst werden. Er sah die philosophische Aufgabe darin, die Lebensmannigfaltigkeit so zu explizieren, wie sie Personen in ihrer Lebensführung schon immer implizit begegnen kann. Diese „naive“ Art und Weise von 
Personen, ihr Leben in der Welt zu führen (Plessner 1975, 27, 331) ${ }^{1}$, wird in der Moderne durch die selbstbewusste Reflexion auf Individualität und die erfahrungswissenschaftlich-technischen Handlungsmöglichkeiten infrage gestellt. Plessners philosophischer Weg, Lebensphänomene anzuschauen, zu verstehen und in ihrem Prozess zu begreifen, wird angesichts dieser Infragestellungen absolviert.

Auf Plessners philosophischem Weg erfolgt mithin eine doppelte Kritik, einerseits an der Naivität einer unkritischen Lebensführung, die sich dem Schein ihrer zufällig eingewöhnten Vorurteile überlässt, andererseits an den überzogenen Ansprüchen, durch reine (lebensfremde) Reflexion und erfahrungswissenschaftliche Bestimmung den Personen ihre Lebensführung abnehmen zu können. Stattdessen zielt Plessner auf eine kritische Artikulation und philosophische Orientierung desjenigen common sense (XIX, XXII) ab, den auch künftig alle möglichen Spezialisten als Laien ihrer Lebensführung miteinander teilen können, wenn sie sich nicht ihrer Personalität und der Qualität ihres Lebens berauben lassen.

Aus der Vielfalt von Plessners auch sozial-, kultur- und geschichtsphilosophischem Lebenswerk interessiert hier im Folgenden seine naturphilosophische Thematisierung von Leben in der unbelebten und belebten Natur. Plessner hatte nicht nur als Philosoph die damals berühmtesten philosophischen Paradigmen vom Neukantianismus (Wilhelm Windelband) über den Neovitalismus (Hans Driesch), die Phänomenologie (Edmund Husserl) und die lebensphilosophisch-geschichtliche Hermeneutik (Wilhelm Dilthey in Georg Mischs Interpretation) bis hin zu dem sozialphilosophischen Begründungsproblem der Soziologie (bei Max und Alfred Weber) durchlaufen. Er war auch Biologe, so dass er in teilnehmender Beobachtung rekonstruieren konnte, welche Qualitäten lebendiger Phänomene von den Biowissenschaften vorausgesetzt werden, wenn sie diese Phänomene durch empirische Reduktion experimentell feststellen und bestimmen. Umso wichtiger war es ihm, bei der Übertragung experimenteller Erfahrungen in die Natur, in andere Handlungsbereiche der Gesellschaft und in die personale Lebensführung empirische Ergebnisse erneut qualitativ in die Lebenssphäre von Personen einzuordnen.

Seine Naturphilosophie Die Stufen des Organischen und der Mensch (1928) enthält am Anfang den Arbeitsplan einer „Neuschöpfung der Philosophie unter dem Aspekt einer Begründung der Lebenserfahrung in Kulturwissenschaft und

1 Im Folgenden beziehen sich alle im Text dieses Kapitels in Klammern gesetzten Seitenzahlen auf Helmuth Plessner (1975): Die Stufen des Organischen und der Mensch. Einleitung in die philosophische Anthropologie [1928], Berlin/New York. 
Weltgeschichte“ (30, zum Arbeitsplan siehe Wunsch 2017) und mündet im Schlusskapitel über Die Sphäre des Menschen (288) in die naturphilosophische Fundierung der Philosophischen Anthropologie, worauf nicht nur „der Mensch“ im Haupttitel, sondern auch der Untertitel des Werks Einleitung in die philosophische Anthropologie verweist. Unter einer Lebenssphäre wird die hybride Einheit von Lebenssubjekt und seiner Gegenwelt verstanden $(66,201,244)$. Angesichts dieses Bogens vom Anfang bis zum Ende des Buches lautet die zentrale Frage: Wie ist das personale Leben von Menschen in die unbelebte und belebte Natur derart gestellt, dass der geschichtliche Aufgabencharakter dieses Lebens verständlich wird? Geistig gesehen ist dieses Leben auf Abstand von Körperbestimmungen (in Raum und Zeit) und auf Distanz zu leiblichen Verhaltensrichtungen (in Raum- und Zeithaftigkeit) gestellt. Dank dieses Abstandes und jener Distanz können Körper und Leibesrichtungen bestimmt werden. Sie werden aus einem geistigen Koordinatensystem (z. B. aus einer Physik und Psychologie) heraus erkannt, das selber ablösbar von den sinnlichen und situativen Eigenschaften der Körper und Leiber ist. Aber zugleich muss sich personales Leben als Leben in Körperlichkeit (vertretbar, austauschbar, ersetzbar) und in Leiblichkeit (unvertretbar, nicht austauschbar, unersetzbar für die Betroffenen) verwirklichen können. Plessner formuliert am Ende seines Buchs Ausdrücke für diese Problemlage in der personalen Lebenssphäre, die berühmt geworden sind: Die exzentrische Positionalität werde durch natürliche Künstlichkeit, vermittelte Unmittelbarkeit (bzw. indirekten Direktheit) und einen utopischen Standort (zwischen Nichtigkeit und Transzendenz) verwirklicht. Seine originäre Lösungsrichtung lautet: Statt in Natur und Kultur, statt in Vermitteltheit und Unmittelbarkeit, statt in Nihilismus und Religion auseinanderfallen zu müssen, kommt es im personalen Leben auf die geschichtliche Verschränkungen dieser Gegensätze im Vollzug an. Darauf komme ich in diesem Kapitel unter 1.6. und ausführlich im 3. Kapitel dies ersten Teils zurück.

Um den Einstieg in Plessners naturphilosophisches Hauptwerk zu erleichtern, kann die Zurückweisung von Erwartungen hilfreich sein, die aus den Philosophien einer absoluten Identität oder absoluten Differenzialität kommen. Plessner denkt kategorial weder die Differenzialität von Phänomenen unter dem Primat ihrer Identität (wie Hegel) noch die Identität von Phänomenen unter dem Primat ihrer Differenzialität (wie Derrida). Vielmehr expliziert er für die Überwindung einer vollständigen Disjunktion (etwas sei entweder materiell oder geistig, entweder physisch oder psychisch) konkrete Drittheiten, also keine „Generalnenner“ allen möglichen Seins (152). Diese Drittheiten werden in der personalen Lebensführung implizit dafür in Anspruch genommen, sich qualitativ orientieren zu können. Sie bezeichnen strukturell einen Bruch (Hiatus, 292), 
der schon immer eine Aufgabe für die Verhaltensbildung von Personen stellt. Seine Überbrückung muss von den Betroffenen in ihrem Tun und Lassen auf eine bestimmte Weise vollzogen werden, ohne dass sie aber die Funktion der Überbrückung in ihrer Generationenfolge für alle Zukunft, d. h. absolut, wirklich festlegen können. Für die von dieser Lage Betroffenen übersteigt die Fraglichkeit, in den Bruch gestellt zu sein, ihre Möglichkeit, darauf abschließend zu antworten, obgleich sie hier uns jetzt zu antworten haben. Daher bleibe personales Leben auch in Zukunft geschichtlich offen, allerdings nicht auf beliebige, sondern die Not des Bruches wendende Weise.

Plessners scharfe Kritik an den Ideologien vom Ende der Geschichte, sei es durch den Dogmatismus der Vernunft, sei es durch den Fanatismus eines Glaubens, auch in einem atheistischen Menschenbild, das den Menschen durch Wissenschaft und Technik zu Gott machen will, ist Kants Einsicht in die Antinomien der reinen Vernunft verpflichtet, aus denen schon immer und stets von Neuem dieser Dogmatismus oder jener Fanatismus einen notwendig scheinhaften Ausweg verspricht. Dabei enthält Plessners ungewöhnliche Figur vom Bruch in der Natur und seiner in Grenzen verwirklichbaren, daher erneut ergänzungsbedürftigen Verschränkung ein Naturverständnis, wie es innerhalb reduktiver Naturalismen und innerhalb des dualistischen Mainstreams der westlichen Moderne nicht möglich ist. Es erfordert eine Kombination aus der phänomenologischen Deskription von Qualitäten der Lebenserfahrung, „Anschauung“ genannt, mit dem hermeneutischen Erschließen, wie verschieden die beschriebenen Lebensphänomene gedeutet werden können, und mit der dialektischen Ordnung von Kategorien, die qualitativ verschiedene Phänomenbereiche im Hinblick auf die Ermöglichung lebendiger Ganzheiten durch Negativität systematisch darstellen. Kurzum: Es handelt sich hier um eine phänomenologische und dialektische Hermeneutik der Natur.

Dieser naturphilosophische Zugang zum Leben ist nicht nur aktuell, weil sich die biomedizinischen Lebenswissenschaften das Thema des Lebens angeeignet haben, während ein großer Teil der Philosophie und Sozial- und Kulturwissenschaften es seit dem linguistic turn in einer Vielzahl weiterer turns geräumt hat. Plessners Naturphilosophie bleibt auch als Alternative zu Heideggers Existenzphilosophie interessant. Heidegger hatte 1927 in seinem Buch Sein und Zeit eine $\mathrm{zu}$ erwartende philosophische Anthropologie vorab dem Primat seiner Fundamentalontologie der Existenzialität unterstellt. Darauf antwortete Plessner im Vorwort seiner Stufen 1928, er könne Heideggers Grundsatz nicht anerkennen,

dass der Untersuchung außermenschlichen Seins eine Existentialanalytik des Menschen notwendig vorhergehen müsse. Diese Idee zeigt ihn [Heidegger: HPK] noch im Banne je- 
ner alten Tradition (die sich in den verschiedensten Formen des Subjektivismus niedergeschlagen hat), wonach der philosophisch Fragende sich selbst existentiell der Nächste und darum der sich im Blick auf das Erfragte Liegende ist. Wir verteidigen im Gegensatz dazu die These - die der Sinn unseres naturphilosophischen Ansatzes und seine Legitimation ist -, dass sich der Mensch in seinem Sein vor allem anderen Sein dadurch auszeichnet, sich weder der Nächste noch der Fernste zu sein, durch eben diese Exzentrizität seiner Lebensform sich selber als Element in einem Meer des Seins vorzufinden und damit trotz des nichtseinsmäßigen Charakters seiner Existenz in eine Reihe mit allen Dingen dieser Welt zu gehören. (V-VI)

Die dann noch Jahrzehnte anhaltende Heidegger-Plessner-Kontroverse trifft das Grundproblem modernen Philosophierens. Wie verhält sich die Frage danach, ein Selbst sein oder nicht sein zu können, zu der Unterscheidung zwischen Eigenem, Anderem und Fremdem? Woher kommt in der westlichen Moderne die Ermächtigung dazu, das eigene Selbst sein zu können, für primär zu halten? Es wird zumeist für so primär gehalten, dass man nicht nur das Andere und Fremde an einem selbst, das nicht selbst sein kann oder soll, zu beherrschen sucht, sondern auch das Andere und Fremde außer dem eigenen Selbst, das Selbst sein kann, sich nicht von selbst zeigen und nicht selbst sein lässt. Charles Taylor unterscheidet heute dieses von Anderem und Fremdem ,abgepufferte Selbst“ von einem für Anderes und Fremdes „porösen Selbst“ (Taylor 2009, 899ff.). Plessner geht im 2. Kapitel der Stufen sehr genau der ontologischen und erkenntnistheoretischen Problemlage nach, die zur dualistischen Verfestigung der Privilegierung des eigenen Selbst führt (siehe Schürmann 2017). Das erkenntnistheoretische Hauptargument dafür bestand in dem zweifellos selbstreferentiellen Charakter personalen Bewusstseins, der im 20. Jahrhundert auf die ebenso zweifellos gegebene Selbstreferenz in der Sprache erweitert wurde. Für Plessner ist spezifisch, dass er die selbstreferentielle „Immanenz des Bewusstseins“ (328-331) gerade für denjenigen Vollzug hält, der die Referenz auf Anderes und Fremdes ermöglicht, statt ins Eigene abzuschließen. Die „Selbsteinsperrung“ ins vermeintlich Eigene und „Selbstaussperrung“ $(48,50)$ vom vermeintlich Andern und Fremden komme durch die Verdinglichung des Bewusstseins zu einem „Kasten“ oder einer „Kammer“ (67) zustande, statt Bewusstsein als den Vollzug der Verschränkung zu begreifen (70f.). Ähnlich gehört für Plessner der idiomatische Selbstausdruck in der Vielfalt der Sprachen zu den geschichtlich pluralen Verwirklichungsbedingungen, um in der Sprache propositionale Aussagen machen $\mathrm{zu}$ können statt diese $\mathrm{zu}$ verunmöglichen (340f.). Mit John L. Austin gesprochen: Performativität ermöglicht Konstativität, statt sie zu vereiteln (Krüger 2001, 1. Kap.). 


\subsection{Die naturphilosophische Originalität der Stufen}

Die berühmte amerikanische Philosophin der Biologie Marjorie Grene hat die naturphilosophische Originalität von Plessners Stufen bereits herausgearbeitet. Sie stellte dieses Werk in eine Reihe mit den Naturphilosophien von Alfred North Whitehead und Maurice Merleau-Ponty. Im Unterschied zu Wissenschaftstheorien der Naturwissenschaften stünden oder fielen die modernen Naturphilosophien des 20. Jahrhunderts mit ihrer Antwort auf die Frage, worin die Spezifik des Lebens im Leben, nicht allein in einem Labor oder in einer Selbstreflexion expliziten Wissens, bestehe. Naturphilosophien stellten das alte Problem, wie lebendige Gegenstände nicht nur im physikalischen Sinne räumlich und zeitlich bestimmt sein können, sondern ihren eigenen Ort und ihre eigene Zeit haben können, auf neue Weise, da cartesianische Entweder-oder-Alternativen an der Lebendigkeit von Gegenständen scheitern (Grene 1966, 252-254). Solche Gegenstände leben nicht dadurch, dass sie entweder psychisch oder physisch sind, sondern indem sie sich verhalten. Lebewesen stellten sich in ihrem Verhaltensvollzug sowohl physisch als auch psychisch dar, weshalb sie eines anschauenden und verstehenden Zuganges bedürfen. Erst in diesem Rahmen würden die Methoden der Reduktion spezifisch lebendiger Phänomene auf physikalische und chemische Tatsachen sinnvoll (Grene 1974, chap. XVI-XVIII).

Das Besondere an Plessners Naturphilosophie zeige sich zunächst darin, so Grene, dass sie den folgenden klassischen Gegensatz überwinde: Leben bestehe entweder aus einem Mechanismus oder aus einer Teleologie der Selbstzweckhaftigkeit. Demgegenüber handele Plessners Naturphilosophie von signifikanten Formen des Lebens, d. h. von unterschiedlichen Arten und Weise zu leben, mithin Leben zu vollziehen. In solchen Formen können mechanische und immanent teleologische Zusammenhänge ineinandergreifen. Merleau-Ponty habe in seiner Leibesphänomenologie diese Orientierung an der signifikanten Lebensform von Plessner indirekt übernommen (Grene 1966, 251). In der Tat verweist Merleau-Ponty in seinen Hauptwerken nicht direkt auf Plessners Stufen, wohl aber auf kleinere Arbeiten Plessners. So übernimmt Merleau-Ponty von Plessner das Verständnis des Leibes als „Umweltintentionalität“ (Merleau-Ponty 1966, 272 mit Verweis auf Plessner 1982c) und die „Verständlichkeit“ des Zusammenhangs zwischen Situation und Reaktion als „Sinn“ (Merleau-Ponty 1976, 148 mit Verweis auf Plessner 1983f). Gleichwohl wird das Defizit von Merleau-Ponty an einer Rezeption von Plessners Stufen durch einen der bekanntesten Neopragmatisten der Gegenwart, Richard Shusterman, bestätigt, wenn er Merleau-Pontys Leibesphänomenologie nicht nur gegenüber cartesianischen Trennungen würdigt, sondern auch dafür kritisiert, dass ihr der reflexive Abstand gegenüber dem Leib vom Standpunkt der „Person“ (293) und ihrer „Mitwelt“ (300-305) 
fehle, den aber Plessner in seinen Stufen entwickelt hat (Shusterman 2008, chapt. 2; Shusterman 2010, 207-212).

Das zweite Besondere an Plessners Naturphilosophie, so Grene weiter, bestehe darin, dass sie einen weiteren alten Gegensatz in der Thematisierung des Lebens überwinde: Leben sei entweder als ein Organismus, der sich selbst organisiert (Organizismus), oder als determiniert durch die Umwelt, an die sich Organismen anpassen und von der sie selegiert werden (Milieu-Theorien), zu begreifen. Diese Überwindung gelinge Plessner dadurch, dass er die Lebendigkeit von Körpern in dem anschaulichen Tatbestand manifestiert sieht, wie solche Körper ihre eigene Grenze realisieren (100-105). Einem lebendigen Körper komme der Übergang zwischen ihm und seinem Medium bzw. seinem Umfeld selbst zu, insofern er sich nach außen und nach innen sowohl öffnen als auch schließen kann. Indem ein Körper dadurch lebt, dass er sich über ihn hinaus und in ihn zurück zu setzen vermag, sich auf diese Weise nicht nur raumhaft, sondern auch zeithaft in seinen Verhaltensrichtungen vorweg und hinterher sein kann (128-130, 176-180), werde von Anfang an das Zusammenspiel zwischen der Binnengliederung des Organismus und seinem Verhaltenspotential in der Umwelt eben als Grenzfrage aufgerollt (Grene 1966, 268). Die Stärke des Ansatzes liege in der Durchführung des Grenzzusammenhanges zwischen den Organisationsformen (Binnengliederungen der Organismen) und den Positionalitätsformen (Verhaltensweisen in der Umwelt) in beide Richtungen, aber auf eine kontingentere Weise, als es die biologische Rede von Anpassung und Selektion gestatte (ebd.). Durch die Öffnung und Schließung der Organisationsformen, durch die Dezentralisierung und Zentralisierung der geschlossenen Organisationsform in Positionalitätsformen werde das seit Aristoteles klassische Thema der pflanzlichen und tierlichen Lebensformen auf neue Weise für die moderne Lebenswelt eingeholt, ohne nämlich einen letzten Beweger oder eine höchste Substanz im Kosmos annehmen zu müssen.

Schließlich treibe Plessner seinen Grundgedanken der je eigenen Grenzrealisierung lebendiger Körper nicht nur durch funktional nötige Korrelationen zwischen Organisations- und Positionalitätsformen hindurch, sondern auch bis in diejenige Grenze hinein, die er eine exzentrische Positionalität (292-295, 300-302) nennt. Damit trete in der Positionalitätsform höher entwickelter Tiere ein Strukturbruch in der leiblichen Konzentrik hervor, d. h. ein Hiatus in der leiblichen Einheit von Organismus und Umwelt, in dem die personale Lebenssphäre von Menschen möglich werden kann. Dieser Bruch in der leiblichen Konzentrik erfordere allerdings, sich aus der leiblichen Einheit des eigenen Organismus mit seiner Umwelt heraus setzen zu können, aber wohin und in welche Zeitlichkeit?: In ein Ex-Zentrum des „Nichts“ (294) an raumzeitlichen 
Bestimmtheiten, in eine „Negativität der Sinne“, die das hier und jetzt Anwesende vom Abwesenden her beurteilt (Grene 1966, 274). Plessner hatte anhand von Wolfgang Köhlers Schimpansen-Versuchen herausgearbeitet, dass es ihrer zweifellos vorhandenen Intelligenz nicht an leiblich positivem Sinn mangele, sondern an dem „Sinn für das Negative“ (270), d. h. an der Leere des Raumes und an der Unerfülltheit der Zeit. Plessners Entdeckung des Hiatus, so Grene, der personales Leben ermögliche, wenn er geistig überbrückt werden kann, werde erst durch eine soziokulturelle Formierung der Mitwelt bestimmbar (Grene 1966, 274).

Damit überschreite Plessners Projekt die Philosophie der Biologie in eine Philosophische Anthropologie für alle möglichen Humanwissenschaften, unter deren Titel Plessner berühmt und zum wichtigsten Konkurrenten von Martin Heidegger geworden ist. Marjorie Grene hatte nach einem Zoologie-Studium selbst 1931-1933 bei Heidegger und Karl Jaspers in Deutschland studiert, sich in den USA über Existentialismus promoviert und unter dem Eindruck von Michael Polanyi eine Wende in die Philosophie der Biologie vollzogen, in der sie sich von Plessner, Adolf Portmann und Erwin Strauss inspirieren ließ. Sie arbeitete der synthetischen Theorie der Evolution (Ernst Mayr) vor, ohne fälschlicher Weise das Wissen als Gewusstes mit seinem realen Gegenstand zu verwechseln (Grene 2002). Die Vermeidung des schlechten Zirkels, auf Grund dieser Verwechselung im Namen der wahren Evolution sprechen zu können, brachte ihr die seltene Ehre ein, dass ihr Werk in der berühmten Library of Living Philosophers 2002 diskutiert wurde.

Umso interessanter ist Grenes frühe Analyse der Gründe dafür, warum Heideggers Existenzialphilosophie zunächst für den Zeitgeist in Kriegszeiten resonanzreicher als Plessners Philosophische Anthropologie sein konnte, sich dies aber auch ändern könne. Was Heidegger seiner dramatischen Reflexion auf die je eigene Existenzialität vorbehalten habe, nämlich dem Schicksal zu trotzen, dem die je eigene Individualität in ihrem Zukunftsbezug auf ihre eigene Sterblichkeit ausgesetzt ist, all dies rekonstruiere Plessner als Strukturmomente, die bereits in den vorpersonalen Lebensformen für eine Dynamik im Lebensprozess als Ganzem sorgen, bevor Personen darüber auch noch als über ihre Eigentlichkeit reflektieren können (Grene 1966, 260, 267). Durch Plessners philosophische Einsicht in die lebendige Natur und unsere Stellung in ihr könne sich die Haltung im Lebensprozess zu ihm im Ganzen ändern. Die persönliche Reflexion auf die je eigene Existenzialität gestalte sich gegenüber anderen viel offener als in Heideggers Sein und Zeit, wenn man mit Plessner einsehe, inwiefern alle Lebewesen schon vorreflexiv ihrer Individualisierung, Sterblichkeit und ihrem Zukunftsbezug im Lebensprozess ausgesetzt sind (Grene 1966, 250, 
276). Die eigene Existenzialität müsse nicht wie bei Heidegger durch die Angst vor dem eigenen Tod entdeckt werden, sondern könne wie bei Plessner im Lachen und Weinen als den Erfahrungen der eigenen Verhaltensgrenzen einsetzen (Plessner 1970).

Plessner beginnt die Stufen mit einem Verweis auf den damals, im ersten Drittel des 20. Jahrhunderts, noch virulenten Zaubercharakter, der im epochal angerufenen Leben zum Ausdruck gekommen (3f.) und in Henri Bergsons Philosophie der kreativen und intuitiven Evolution ausformuliert worden sei (8f.). Plessners phänomenologisch anschauender und hermeneutisch verstehender Zugang zum Lebendigen zielt auf die philosophische Erkenntnis der irreduziblen Qualitäten in der Erfahrung des Lebendigen, die selbst eine lebendige ist, ab. Er, der selbst auch Zoologe war und lange mit dem niederländischen Verhaltensforscher Frederik Jacobus Johannes Buytendijk zusammengearbeitet hat, bejaht, dass der biologisch-naturwissenschaftliche Zugang empirisch durch Methoden der Reduktion solcher Qualitäten auf messbare und feststellbare Tatsachen erfolgt, da so präzise reproduzierbare und verwertbare Erfahrungserkenntnisse entstehen. Daraus ergebe sich aber gerade die Aufgabe einer „Kooperation“ (70) zwischen den Biowissenschaften und der Philosophie der lebendigen Natur, in der nicht die eine Seite die andere ersetzen kann. Auch Biologen bewältigen ihre personale Lebensführung nicht durch ihre reduktiven Methoden. Sie setzen eine qualitative Spezifik lebendiger Kontexte nicht nur in ihrem Leben, sondern auch in ihrem Beruf voraus, um sinnvoll reduzieren zu können. Ebenso könne es nicht umgekehrt die Aufgabe der modernen Naturphilosophie sein, unabhängig von und vor jeder biowissenschaftlichen Erfahrungsart die Wesensspezifik lebendiger Phänomene rein spekulativ aus metaphysischen Annahmen deduzieren $\mathrm{zu}$ wollen. Vielmehr habe eine solche naturphilosophische Deduktion sowohl im Hinblick auf die biologisch fassbare Erfahrungsart (Ontik) als auch unter dem Gesichtspunkt der Realisierung der angeschauten Qualitäten (122) zu erfolgen. Diese Deduktion sei so zu leisten, dass sich zwischen der empirisch nötigen Einschränkung auf Mess- und Feststellbares einerseits und der angeschauten Qualität der lebendigen Phänomene andererseits diejenige Ontologie des Lebendigen erschließen lasse, die den anschaulichen Tatbestand wirklich machen, also von einem bloßen Schein unterscheiden kann (118, 126; vgl. Edinger 2017).

Dabei sei zwar das Einschalten der vorwissenschaftlichen, sogenannt naiven Weltsicht und Lebenserfahrung, die alle möglichen Spezialisten als Laien ihrer Lebensführung miteinander im common sense teilen, hilfreich, aber auch nicht das letzte Wort in der Sache (XXII, 26, 51, 118). Die Naturphilosophie lege in ihrem Kategoriennetz jene irreduziblen Erfahrungsqualitäten frei, die sich als 
die bleibenden „Vorbedingungen“ bzw. nicht ableitbaren „Voraussetzungen“ der Forschung und der Lebensführung auch künftig erweisen können (72, 88, 114, 301). Daher unterscheidet Plessner klar philosophische Kategorien von empirischen Begriffen. „Kategorien sind keine Begriffe, sondern ermöglichen sie, weil sie Formen der Übereinstimmung zwischen heterogenen Sphären, sowohl zwischen Denken und Anschauen wie zwischen Subjekt und Objekt, bedeuten“ (116, vgl. 65f.).

\subsection{Die Theorie der weltoffenen Fraglichkeit personalen Lebens und ihre Methodenkombination}

Auf das geschichtsphilosophische Thema einer offenen Zukunft, das Plessner ausdrücklich gegen alle Ideologien von einem Ende der Geschichte vertritt, kommt er in seinem Buch Macht und menschliche Natur. Ein Versuch zur Anthropologie der geschichtlichen Weltansicht (1931) ausführlich zu sprechen, in dem er das Prinzip der Unergründlichkeit des auch künftig geschichtlichen Lebens für seine Philosophische Anthropologie als verbindlich erklärt (Plessner 1981d, 161, 181-184, 222). Seine Begrenzung der Verabsolutierung positiver Erkenntnisse, ganz gleich, ob sie aus der Erfahrungswissenschaft oder aus der metaphysischen Spekulation kommen, ist jedoch auch schon in den Stufen in der Kritik an dem traditionellen „Generalnenner“ für „alles Sein“ präsent (152), weshalb es sich für die Lektüre empfiehlt, die Plessner originäre Integration von Phänomenologie, Hermeneutik und Dialektik als Methoden, nicht als abschließende Theorien im Auge zu behalten. Plessners Theorie steht und fällt mit dem Offenhalten der Fraglichkeit des Menschen. In aller Gemeinsamkeit personalen Lebens mit non-personalen Lebensformen unterscheide es sich von diesen in der Erschließung einer Weltoffenheit, aus der heraus die Schaffung der eigenen Umwelt zur stets ergänzungsbedürftigen Aufgabe werde (293f., 310f.). Personales Leben nimmt öffentlich die „Negativität des Absoluten“ als seine Zukunft ermöglichende Funktionsstruktur in Anspruch. Insofern öffentlich keine positive Ausmalung eines bestimmten Absoluten für alle und ewig vorgeschrieben werde, kann privat die Freiheit der Religionen und Weltanschauungen in ihrer Pluralität ermöglicht werden. In der gottgleichen Realisierung eines positiven Absoluten für alle fiele sich demgegenüber das personale Leben selbst zum Opfer (Krüger 1999, 30-32, 266; Krüger 2001, 108f., 154f.; Krüger 2006c, 26-29). Diese theoretisch konsequente Ausrichtung an der öffentlichen (nicht privaten) Negativität des Absoluten und damit einer offenen Fraglichkeit teilt keine andere der genannten philosophischen Richtungen, die daher oft durch Unverständnis oder falsche Erwartung auf Plessners Instrumentierung ihrer Metho- 
den zu einem anderen als dem ihnen geläufigen Zweck reagiert haben. So fehlte Hans-Georg Gadamer, in dessen Hermeneutik Sein, das verstanden werden kann, Sprache ist, der Zugang zur Thematisierung des Menschen in der lebendigen Natur (Gadamer 1990, 448-460; 478), als ob sich Menschen nicht verhalten würden. Jürgen Habermas (in seiner mittleren Schaffensperiode) fehlte bei Plessner die Ableitung der Spezifik des Menschen aus der sprachlichen Intersubjektivität, die man auch als den normativen Maßstab des geschichtlichen Fortschritts verwenden könne, so als gäbe es doch ein Telos der Geschichte (Habermas 1987, 138-140).

Plessners Philosophische Anthropologie konnte tatsächlich nicht solche sprach- und geschichtsidealistischen Ansprüche befriedigen, ebenso wenig die szientistische Auffassung, durch erfahrungswissenschaftliche Reduktionen einer freien Gesellschaft die Lösung ihrer Probleme vorschreiben zu können. Reduktive Naturalismen verfallen oft dem schlechten metaphysischen Zirkel, sich selbst für die beste aller selegierten Anpassungen der Natur zu halten, d. h. „die Natur wird bei dieser Erklärung bereits als das vorausgesetzt, was erst kraft der Kategorien möglich“ werden soll (7). Plessners Philosophische Anthropologie dient dagegen der öffentlichen Beratung in einer pluralen Gesellschaft, in der niemand den Anderen ihre personal freie Lebensführung abnehmen kann. Seine theoretisch originäre Integration der folgenden vier philosophischen Methoden (Krüger 2006a;) - nimmt man nur öffentlich an ihr teil - hilft, durch Skepsis wider ein positives Absolutum für alle und ewig (346) selbst in der Lebensführung mündig und urteilsfähig zu werden (Krüger 1999; Schürmann 2014):

A) In der Thematisierung geschichtlicher Lebenserfahrung ist es nötig, den qualitativen Anschauungscharakter dieser Erfahrung zu erfassen, der in seiner Fülle über dasjenige hinausgeht, was man nach erfahrungswissenschaftlichen Schemata von der Erfahrung feststellen und messen $(78,118 f$.) kann. Plessner funktioniert daher die Phänomenologie für die Rekonstruktion der Anschauung des Qualitativen in der Lebenserfahrung methodisch um, ohne theoretisch in Edmund Husserls Dualismus zurückzufallen (V, 28, 30, 118f.). Plessner bleibt aber der Devise verpflichtet, dass sich eine ursprüngliche Qualität von Lebenserfahrung für diejenigen, die von ihr in ihrer Lebensführung betroffen sind, nicht einfach ersetzen oder ungeschehen machen lässt, indem diese unmittelbare Qualität aus einer anderen Erkenntnis abgeleitet wird oder als vermittelt reflektiert wird. Leben fügt sich keinem solchen intellektuellen Fehlschluss. Liebe und Kunstgenuss sind nicht ersetzbar, und wie schwer es wird, mit einem Trauma umzugehen, davon berichtet jede darauf bezogene Psychotherapie (vgl. 37). 
B) Fragliche Anschauungen können im Verhalten verschieden verstanden und dem gemäß verschieden beantwortet werden, was $\mathrm{zu}$ verschiedenen geschichtlichen Konsequenzen führt. Plessner schließt in dieser Hinsicht an Wilhelm Diltheys lebensphilosophisch-historische Hermeneutik an, wie sie von Georg Misch systematisiert wurde (Schürmann 2011, 4. Kap.), von dem er das Prinzip der Unergründlichkeit des geschichtlichen Lebens übernimmt. Hier handelt es sich nicht wie bei Gadamer um eine sprachzentrierte, sondern auf alle möglichen Verhaltensweisen des Ausdrucks und Verstehens erweiterte Hermeneutik (23), in die Plessner die interkulturell universellen Ausdrucksweisen des Lachens und Weinens (Plessner 1982f) einbringt. Sie führt auch aus dem bloßen Relativismus aller Kulturepochen und Soziokulturen von Menschen heraus, denn die jeweilige historische Relativität, insbesondere die des Westens, wird auf eine weltgeschichtlich gemeinsame und offene Zukunft bezogen und dafür neu bewertbar (vgl. Krüger 2013a, im vorliegenden Buch III. Teil).

C) Was man in der personalen Lebensführung (phänomenologisch) angeschaut und (hermeneutisch) verstanden hat - diesen Zusammenhang von Frage und Antwort -, gilt es in der Philosophie kategorial zu explizieren (113-114). In den philosophischen Kategorien wird dieser Zusammenhang derart rekonstruiert, dass einerseits die erfahrungswissenschaftlich eingeschränkte Interpretation von Erfahrung ermöglicht wird, andererseits aber zugleich die Qualität der Lebenserfahrung für die personale Lebensführung eine unvertretbare Aufgabe bleibt. Daher ist Plessners „Deduktion“ eine „doppelseitige“ (Mitscherlich 2007, Mitscherlich-Schönherr 2017). Plessner schließt in der Systematisierung der Kategorien an Formen Hegelscher Dialektik $(115,305)$ an, wie sie Josef König in seinem Verständnis der Intuition als einer Verschränkung (Schürmann 2011, 4.3., 5.2.) und Nicolai Hartmann in dem Stufenbau seiner Neuen Ontologie (Wunsch 2015) entwickelt haben, ohne Hegels systematischer Aufhebung in ein positiv wissbares Absolutes zu folgen. Plessners offene Fraglichkeit transformiert statt eines solchen Endes der Geschichte seit seiner Habilitationsschrift (Plessner 1981e) von 1920 Kants Agnostizismus (in der Behandlung der Antinomien reiner Vernunft) in eine Negativität des Absoluten, um nicht wie die meisten seiner Zeitgenossen einem ideologischen Fanatismus oder Dogmatismus anheim zu fallen.

D) Die kategoriale Rekonstruktion von „Lebenserfahrungen“ (28, 30, 37) legt in ihrer systematischen Vernetzung zu einem offenen Ganzen frei, was man für diese qualitativen Erfahrungen an Ermöglichungsstrukturen und Ermöglichungsfunktionen in Anspruch genommen hat. Dieses Verfahren folgt einem „quasi-transzendentalen“ Philosophieverständnis (Krüger 2001, 30f., 44, 48, 88f., 92f., 289f.; Krüger 2006a, 204-212). Es ist insofern noch transzendental, als 
es die Aufgabe der Philosophie in der Rekonstruktion der Ermöglichungsbedingungen von Erfahrung sieht. Aber es ist nur quasi transzendental, insofern es sich nicht mehr wie bei Kant auf die naturwissenschaftliche Erfahrungsart beschränkt, sondern auf geschichtliche Lebenserfahrung umgestellt wird. Auch die Antwort auf die Frage nach den Ermöglichungsbedingungen wird nicht mehr auf das Selbstbewusstsein begrenzt, sondern auf die Strukturen und Funktionen der Ermöglichung geschichtlicher Lebensprozesse erweitert. Berücksichtige man andere Lebensformen als humane in der Natur, brauche Bewusstsein „nicht Selbstbewusstsein zu sein“ (67). „Auf jeden Fall brauchen Bedingungen der Möglichkeit der Erfahrung nicht Erkenntnisbedingungen zu sein. Es kann auch um die Möglichkeit von Gegenständen und Substraten, an denen die Erfahrung ansetzt, gestritten werden“ (75).

Plessners Werk Die Stufen des Organischen und der Mensch enthält einen "Arbeitsplan“ (26) für die Neuentwicklung der Philosophie und die naturphilosophische Fundierung seiner Philosophischen Anthropologie. Der Plan folgt dem Zweck der „Neuschöpfung der Philosophie unter dem Aspekt einer Begründung der Lebenserfahrung in Kulturwissenschaft und Weltgeschichte“ (30). Analog sei Kant sein Ziel, den Weltbegriff von Philosophie, unter dem Aspekt der naturwissenschaftlichen Erfahrung angegangen, um den Weg der Vernunftkritik beschreiten zu können. Die Etappen auf Plessners Weg und sein neues Mittel sind: „Grundlegung der Geisteswissenschaften durch Hermeneutik, Konstituierung der Hermeneutik als philosophische Anthropologie, Durchführung der Anthropologie auf Grund einer Philosophie des lebendigen Daseins und seiner natürlichen Horizonte; und ein wesentliches Mittel (nicht das einzige), auf ihm weiterzukommen, ist die phänomenologische Deskription“ (30). Der Aspekt, unter dem diese Etappen durchlaufen werden, beinhaltet die folgende neue Fokussierung: „In seinem Mittelpunkt steht der Mensch. Nicht als Objekt einer Wissenschaft, nicht als Subjekt seines Bewusstseins, sondern als Subjekt und Objekt seines Lebens, d. h. so, wie er sich selbst Gegenstand und Zentrum ist“ (31).

Die Frage, inwiefern zwischen dem Menschen und dem Lebenshorizont der Welt eine „Wesenskoexistenz“ und nicht allein Zufall sich einspielen kann, sei in unserer kulturellen Lage in zwei Richtungen zu erforschen. Sie kann ,horizontal“ aufgerollt werden, d. h. in geschichtlicher Richtung auf die „Taten und Leiden“ des Menschen „als Subjekt-Objekt der Kultur“, oder „vertikal, d. h. in der Richtung, die sich aus seiner naturgewachsenen Stellung in der Welt als Organismus in der Reihe der Organismen“, also als „Subjekt-Objekt der Natur“ (32) ergibt. Die horizontale Untersuchungsrichtung war Plessner in kulturellsymbolischer Hinsicht bereits in seinem Buch Die Einheit der Sinne. Grundlinien 
einer Ästhesiologie des Geistes (1923) und im Hinblick auf Soziales in seinem Essay Grenzen der Gemeinschaft. Eine Kritik des sozialen Radikalismus (1925) angegangen, worauf er in geschichtlich-politischer Hinsicht 1931 in Macht und menschliche Natur. Ein Versuch zur Anthropologie der geschichtlichen Weltansicht und der dazu gehörigen Fallstudie Die verspätete Nation (1935/1959) über die Rolle Deutschlands in Europa zurückkam. Die wichtigste Vorarbeit zum vertikalen Vergleich, der in den Stufen durchgeführt wird, bestand in dem grossen Aufsatz Die Deutung des mimischen Ausdrucks. Ein Beitrag zur Lehre vom Bewusstsein des anderen Ichs (1925), in dem Plessner gemeinsam mit Buytendijk die Leiblichkeit des Verhaltens als Ausweg aus dualistischen Fehlalternativen freilegte, woran Merleau-Ponty, wie oben bereits erwähnt, seine Leibesphänomenologie angeschlossen hat. Die wichtigste Ergänzung der Stufen stellt das Buch Lachen und Weinen (1941) dar, da es die exzentrische Positionalität nicht mehr nur in dem Ansprechen der zeitgenössischen Leserinnen als Menschen (ad hominem), sondern anhand dieses Anschauungsbestandes von Grenzerfahrungen personaler Lebewesen erläutert (vgl. zum systematischen Zusammenhang in Plessners Gesamtwerk Krüger 1999 und Einführung in das vorliegende Buch).

Der vertikale Vergleich humaner mit non-humanen Lebensformen wird auf dem Wege der Naturphilosophie fundiert. Sie rekonstruiert drei große Probleme, in deren Ermöglichungsrahmen die Biowissenschaften ihre reproduzierbaren Erkenntnisleistungen erbringen: Erstens: Wie lassen sich belebte Körper von unbelebten Körpern unterscheiden? Diese Frage betrifft den Übergang von WasFragen zur Ermöglichung von Wer-Fragen, die spezifisch lebendige Phänomene unterstellen. Lebewesen sind noch keine Personen, aber sie haben bereits einen eigenen Verhaltensspielraum, der in unserer Lebenswelt etwas anrichten kann. Diese Frage rekonstruiere ich im folgenden Unterkapitel 1.4. Zweitens: Wie lassen sich innerhalb der lebendigen Natur Organisations- und Positionalitätsformen unterscheiden? Wie kann es also zu einem Zusammenspiel zwischen den Binnengliederungen der Organismen und ihren Verhaltensweisen in der Umwelt kommen? Dieser Frage gehe ich hier unter 1.5. nach. Drittens: Wie lassen sich personale Lebensformen, die in einer exzentrischen Positionalitätsform ermöglicht werden, von zentrischen Positionalitätsformen unterscheiden, die tierliche Lebensformen ermöglichen? Dieses Problem betrifft eine Einschränkung der Wer-Fragen nach den Lebewesen im Lebensprozess auf den Mitvollzug von Personalität in dem Geist einer Mitwelt, der von ihren Fellows geteilt werden kann. Diese Fragestellung wird unter 1.6. entwickelt. 


\subsection{Von der Realisierung der eigenen Grenze in der Positionalität zum Prozess- und Entwicklungscharakter des Lebendigen}

Wenn man sich fragt, wie die Anschauung von bestimmten unbelebten Körpern in Raum und Zeit gelingen kann, dann scheint das angeschaute Ding aus einem Kern zu bestehen, der einen Mantel von Eigenschaften trägt. Was man hier und jetzt wahrnimmt, sind diese empirischen Eigenschaften, die man im Gegensatz zu anderen Eigenschaften bestimmen kann. Sie können im Nacheinander der Zeit und im Nebeneinander des Raumes wechseln, ohne dass sich der Dingkern als die Einheit des Dinges auflösen müsste. Täte er dies, handelte es sich um eine andere Dingart. Irgendwie müssen die Dingkerne und ihre Eigenschaftsmäntel nach Arten miteinander zusammenhängen. Der Kern scheint vom Inneren seiner Eigenschaften her, die wir von außen beobachten können, ihre bestimmte Einheit zu tragen. Sie kann sich in Gestaltsprüngen (stehendes Wasser gefriert) und Artsprüngen (aus der Telefonanlage wird Skype) komplizieren, ohne dass man für den Wechsel zwischen Kern und Eigenschaften andere als im lebensweltlichen Sinne mechanische Kräfte erwarten würde.

Fragt man sich aber weiter, wie die Anschauung von bestimmten belebten Körpern in Raum und Zeit gelingen kann, geschieht in einer solchen Anschauung mehr als ein Gestalten- und Artensprung innerhalb des Unbelebten. Lässt man dem belebten Körper Raum und Zeit, damit er sich zeigen kann, entfaltet sich in dem Wechsel zwischen seinem Kern und seinen Eigenschaften eine innere Dynamik, die sich auch in seinem Äußeren auf eine übermechanisch ganzheitliche Weise entfaltet. Sie greift sowohl nach innen als auch nach außen Platz und ufert aus in ihrer Zeit. Die ganzheitliche Dynamik des Lebendigen behauptet sich als Eigenraum, was Plessner Raumhaftigkeit, und Eigenzeit, was Plessner Zeithaftigkeit nennt. Dabei hört diese Dynamik nicht auf, an die Bedingtheiten der unbelebten Körper gebunden zu bleiben, so im Stoffwechsel und ihrer Abhängigkeit von Medien in ihrer Umgebung. Wie lässt sich dieser, lebensweltlich nicht ersetzbare Sprung in der Anschauung von belebten gegenüber unbelebten Dingen einsichtig machen?

Für die Beantwortung dieser Frage entwickelt Plessner seine Hypothese, die Spezifik der Lebendigkeit von Körpern bestehe darin, dass ihnen nicht nur wie den unbelebten Körpern Konturen als eine von außen beobachtbare Grenze zukommen, sondern dass sie ihre eigene Grenze realisieren. Die Bewegungsrichtungen zwischen unbelebten Körpern in Raum und Zeit finden in bestimmten Konturen Anhaltspunkte für die Unterscheidung der Körper nach Dingarten. Relativ auf einen bestimmten unbelebten Körper bezogen lassen sich die diver- 
genten Richtungen nach außen und nach innen anhand seiner Konturen differenzieren. Diese Unterscheidung ist aber relational umkehrbar auch für alle anderen unbelebten Körper zu treffen. Die Bewegungsrichtungen und ihre Relationen gehen durch alle Körper hindurch. Unbelebte Körper hören da auf, wo andere Körper oder Medien ihrer Umgebung beginnen, und sie beginnen da, wo andere Körper oder Medien ihrer Umgebung aufhören. Das „Zwischen“ zwischen ihnen ist „leer“ im Sinne des leeren Raumes und der leeren Zeit (103f.). Es gehört weder dem Körper noch dem Medium an, sofern es sich aus den Koordinaten einer unterstellten Raumzeit als dem Rahmen ergibt. Dieses Dazwischen gehört aber im Sinne mechanischer Gesetze beiden, Körper und Medium, an, insoweit diese Regelmäßigkeiten sowohl in den Körpern als auch in den Medien ihrer Umgebung wirken. Unbelebte Körper bilden Konturen aus, die mechanischen Gesetzen unterliegen, aber diese Konturen dienen nicht der Funktion, den jeweiligen Körper im Ganzen, d. h. übergestalthaft und übersummenhaft, zu begrenzen.

Stehen Konturen aber in diesem ganzheitlichen Funktionszusammenhang, dann sind sie nicht nur im Sinne mechanischer Bewegungsrichtungen Anhaltspunkte dafür, wo und wann der unbelebte Körper anfängt und aufhört. Gehört der Rand des Körpers zu seiner übergestalthaften und übersummenhaften, $d . h$. ganzheitlichen, gibt es ein Außen und Innen des betreffenden Körpers so, dass Außen und Innen ihm zugehören. Er staut und lockert sich nach innen und verhält sich nach außen. Im Falle der Realisierung der eigenen Grenze schließt sich der Körper gegen das ihm Äußere, das „ihm entgegen ist“ (127f.), ab und auf. Gleichursprünglich verschließt und öffnet er damit sein Inneres, dem er auch von außen zurückkommend entgegen ist.

Ein Körper, der seine eigene Grenze verwirklicht, vollzieht selbst den Grenzübergang zwischen seinem Inneren und Äußeren in beide Richtungen, dem Sinne nach sowohl von innen nach außen als auch von außen nach innen (103f.). Er geht über sich hinaus ins Äußere und kommt von dort zu sich zurück, kurz: er positioniert sich in seiner Umgebung. Und er geht gleichursprünglich in sich hinein ins Innere und kommt von dort zurück ins Äußere, kurz: er organisiert sich. In seinem „positionalen Charakter oder seiner Positionalität“ bestimmen „die Momente des ,über ihm Hinaus' und das ,ihm Entgegen, in ihn Hinein“ ein spezifisches Sein des belebten Körpers, das im Grenzdurchgang angehoben und dadurch setzbar wird“ (129). „In den spezifischen Weisen ,über ihm hinaus“ und ,ihm entgegen“ wird der Körper von ihm abgehoben und zu ihm in Beziehung gebracht, strenger gesagt: ist der Körper außerhalb und innerhalb seiner. Der unbelebte Körper ist von dieser Komplikation frei“ (ebd.). Der belebte Körper ist nicht nur ein „bloßes Ding, sondern ein Wesen“, das auch für sich ist, 
eben ein „Lebewesen“ (131). Es verhält sich zu der Stelle, an der es sich im leeren Raum befindet, „raumhaft“, indem es seinen Raum auf ihm unumkehrbare Weise erfüllt und behauptet. Es verhält sich zu der Gegenwart, in der es sich in der leeren Zeit befindet, „zeithaft“, indem es diese Gegenwart auf ihm irreversible Weise erfüllt und behauptet (vgl. 132, 175-177).

Ein Körper, der sich (dem Sinne nach von innen und nach innen) organisiert und (von außen und nach außen) positioniert, hört nicht auf, auch ein anorganischer Körper zu sein. Insofern er im spezifizierenden Sinne lebt, muss er dasselbe Wesen bleiben können in allen möglichen Grenzübergängen. Insofern er auch aus anorganischen Körpern besteht, muss er das gleiche bleiben, d. h. eine Kombination anorganischer Körper, die für das lebendige Ganze funktional äquivalent ist. Insoweit er dasselbe und das gleiche bleibt, führt er Tautologien auf. Diese müssten aber, im Sinne einer lebensnötigen Möglichkeit in anderer Hinsicht mit Paradoxa zusammenfallen. Dasselbe ist nicht das Gleiche und umgekehrt. Das Äußere ist nicht das Innere und umgekehrt. Das Lebendige ist nicht das Anorganische und umgekehrt. Die Bewegungsrichtungen bleiben divergent und reversibel, auch wenn sie im Vollzug des Lebewesens dem Sinne nach konvergieren und unumkehrbar werden. „Bleiben, was es ist, Übergehen in das, was es nicht ist (über ihm hinaus) und in das, was es ist (in ihm hinein)“ (138).

Wie könnte es möglich sein, dass sich diese Tautologien und jene Paradoxa im Raum und in der Zeit raumhaft und zeithaft ereignen können? - Indem sie sich im Raum und in der Zeit auf verschiedene Stellen verteilen, die raumhaft und zeithaft durch Organisation und Positionierung integriert werden. Unterhalb dieser Komplexion zum Ganzen im Prozess ist es unmöglich, Leben verständlich werden zu lassen: „Ein Ding positionalen Charakters kann nur sein, indem es wird; der Prozess ist die Weise seines Seins“ (132).

Insofern in einem solchen Prozess das Ausgangsetwas zu einem Endetwas wird, ist er gerichtet: „Die Synthese findet als eine besonders gerichtete Form des modus procedendi statt: als Entwicklung“ (140). Um diese Entwicklungsrichtung des Prozesses zu verstehen, überzeugt die Annahme nicht, es gehe nur um den Modus eines bloßen „Vermögens und Könnens“, die Kannqualität des „Könnens oder Nicht-Könnens“: Dann denkt man noch dualistisch, zu der Seinsweise des Körpers komme aus unserem Selbstverständnis ein Können oder Vermögen irgendwie hinzu. „Kannqualität als Seinsqualität, seiende Möglichkeit gilt es zu begreifen“ (172). Diese seiende Möglichkeit besteht nicht in der Art und Weise von Möglichkeit, die man mit dem Lebewesen machen kann (,Mitihm-Möglichkeit“), sondern in einer Art und Weise von Möglichkeit, die an dem 
lebenden Körper selbst vorkommt, in einer „An-ihm-Möglichkeit des physischen Dinges“ (174).

Wolle man dieses Möglichsein unabhängig von unserer eigenen deterministischen Teleologie (178) verstehen, bezeichne es nur eine „besondere Richtung vom Nichtsein zum Sein, die in dem Wort Noch Nicht festgehalten wird“ und die Einheit des Körpers in der Zukunft betrifft:

Möglichkeit fasst also eine Richtungseinheit, die gegen die Bestimmtheitsrichtung des Seienden in der Zeit Vergangenheit Gegenwart Zukunft gekehrt ist. Im Können des Seins wird letztlich nichts anderes als ein Vorwegverhältnis statuiert, in welchem die Abhängigkeitsrichtung von der Zukunft zur Gegenwart läuft. (176)

Dafür muss man annehmen, soll es mit der Determinationsrichtung verträglich bleiben, dass zwischen den Erfüllungen der Zeitmodi ein „zeithaftes“ Verhältnis stattfinde, das nicht innerhalb der leeren Zeit liege, also von ihrem Standpunkt als „zeitlos“ erscheint: „Erst dem lebendigen Sein ist Zeit in ihren Modis wesenhaft, denn es konstituiert sich vermittels der Zeit, insofern es ein Sein bedeutet, das ihm selbst vorweg ist“(179). Es werde nicht ein Bestimmtes vorweggenommen, das erst noch kommen werde, sondern ein Selbstbezug, der als Erfüllung der Zukunft in der konkreten Gegenwart bestimmt ist. „Das ,Ihm selbst Vorweg' und das lebendige Sein besagen ein und dasselbe. Also ist lebendiges Sein ebenso sehr ihm selbst nach oder Erfüllung seiner selbst“ (180). Es lebt im Modus seiner Fundierung aus der Zukunft in seiner konkreten Gegenwart, d. h. im Modus seiner „erfüllten Potentialität“ (ebd.).

\subsection{Organisations- und Positionierungsweisen des Lebendigen im Leben als Ganzem und der Unterschied zwischen offener und geschlossener Organisationsform}

Häufig wird der lebendige Körper als der Träger des Lebens mit dem Leben als dem Ganzen des Prozesses und der Entwicklung verwechselt. Dann wird das Ganze des Lebensprozesses, auf das die Entwicklungsrichtung am lebendigen Körper zeigt (s. o.), übersehen, häufig deshalb, weil es keine „Mit-Möglichkeit“ im Wozu unserer Teleologie (s. o.) ergibt. Das Ganze des Lebensprozesses wird dann auf die Organismushaftigkeit des lebendigen Trägers in sich verkürzt, wie auf dem Bilde vom Leviathan, als ob dieser Träger je autark sein könnte. Plessner folgt dagegen dem „Über-ihn-Hinaus“ und dem „Ihm-Vorweg“ des lebendigen Körpers in den Lebenskreis: „So entsteht der Kreis des Lebens, dessen eine Hälfte vom Organismus, dessen andere vom Positionsfeld gebildet 
wird“ (192). Im Lebenskreis ist der lebendige Träger „eben Teil, ergänzungsbedürftig, seine Autarkie ist dahin. Autonom bleibt er, weil nichts an ihn herankommt und nichts auf ihn und in ihm Einfluss gewinnt, das er nicht dem Gesetz des begrenzt-grenzhaften Systems unterwirft“ (193). Gegen die Betrachtung, als ob sich der Organismus auch vorbewusst Selbstzweck sein müsste, lässt sich die Frage stellen, ob er nicht mindestens auch ein „Mittel zum Leben wird“, das ihn und seine Art und Weise zu leben überschreitet in ein „Hinaussein, durch welches er wieder zu ihm zurückgeleitet wird, in welchem er sich zur Einheit des Ganzen vermittelt“ (191). „Der Organismus ist Einheit nur als durch Anderes, als er selbst ist, in ihm vermittelter Körper, Glied eines Ganzen, das über ihm hinausliegt“ (195). Der Konflikt zwischen lebendigem Körper und dem Lebensprozess im Ganzen kann nicht kategorial vorentschieden werden zugunsten entweder innerer Organismushaftigkeit oder innerer Selbstzweckhaftigkeit, die es beide ohne einen Lebenskreis nicht geben kann.

Wenn man an der Hypothese festhält, der lebendige Körper realisiere seine eigene Grenze, dann bedeutet der Vollzug des Grenzüberganges sowohl Abschluss als auch Aufschluss des lebenden Körpers gegenüber seinem Umfeld. Schließung (in sich hinein und sich entgegen) und Öffnung (über sich hinaus und anderem entgegen) müssen der lebensnötigen Möglichkeit nach in Raum und Zeit so verteilt werden, dass sie raum- und zeithaft im Prozess zusammengeführt werden können, nämlich einer Entwicklungsrichtung nach. Der lebende Körper kann sich „gleichsinnig“, d. h. „umkehrbar“, mit seinem Positionsfeld und „gegensinnig“, d. h. „nichtumkehrbar“ (204), gegen dieses Feld verhalten. Seine Art und Weise, sich nach innen und von innen zu organisieren, und seine Art und Weise, sich nach außen und von außen zu positionieren, müssen zu einander passen, insofern er lebt. „Er muss ins Medium passen und zugleich Spielraum in ihm haben, um nicht nur innerhalb der festen Harmonieformen, sondern mit ihnen Gefahren zu bestehen“ (205). Was den Stoffwechsel und den Energieaustausch angeht, werden so die Teilprozesse der Assimilation und Dissimilation nach der Grenzhypothese verständlich. „Nur unter der Bedingung des inneren Zerfalls in gegensinnig zueinander gerichtete Prozesse ,öffnet‘ sich das in seinen Grenzen als Eigensystem unbedingt geschlossene lebendige Körperding den Einwirkungen von außen und nach außen“ (200).

Aber diese physikalisch-chemische, in der Allgemeinbildung lebensweltlich gewordene Einsicht klärt noch nicht die Frage, wie der „Hiatus“ zwischen dem Organismus und der Umgebung „nicht zerstört, sondern überbrückt“ (204) bzw. „ausgeglichen, nicht aufgehoben“ (200) wird. Man missverstehe die Redeweise von der „Anpassung“, wenn man sie entweder nur für eine hier und jetzt zu leistende „Anpassung“ (im Sinne eines akuten Handlungszwanges) oder nur für 
eine schon vorgegebene „primäre Angepasstheit“ (im Sinne bewährter Strukturen und Funktionen) halte. Gäbe es nur letztere, d. h. eine strukturfunktionale Vorangepasstheit des Organismus, dann schrumpfte das Positionsfeld auf den natürlichen Ort des lebenden Körpers zusammen, in dem er sich wie ein Solipsist in seiner „absoluten Immanenz“ bewegen würde. Handelte es sich umgekehrt allein um akute und daher permanent zu repetierende Anpassung, unterstellte man, der Organismus befände sich nicht in seinem Positionsfeld, sondern in einer ,Zone vollkommener Fremdheit, Unvorhersehbarkeit und Unabhängigkeit“, mithin „isoliert und preisgegeben an eine absolute Transzendenz“ (203). Statt in diesem Entweder-Oder werde Leben in einem anderen Spektrum des aufeinander Einspielens ermöglicht:

Insofern das Verhältnis der Anpassung (Angepasstheit) ein labiles, $d . h$. ein der ,Form nach in seinem Gelingen vorgegebenes, dem ,Inhalt' nach aber ein gelingendes oder misslingendes ist, bleibt der Organismus bei aller Geborgenheit gefährdet. Das Positionsfeld oder das Milieu ist wesensmäßig Schauplatz von Kämpfen und Sphäre des Schutzes. [...] Vorgreifend in der Form, suchend im konkreten lebendigen Akt nach Kontakt mit dem Medium, angepasst-anpassend spielt sich das Leben des Organismus im Verhältnis zum Umfeld ab. (207)

Eine philosophisch ähnliche Revision wie im Verständnis der „Anpassung“ strebt Plessner auch mit der Redeweise von der „Selektion“ an. Rückte das Reden von der Anpassung die Anforderungen an den Organismus in den Fokus, um das riskante Zusammenspiel zwischen ihm und der Umwelt erklärlich werden zu lassen, so schaut man in der Redeweise von der Auswahl auf dieses Spiel mit Rücksicht aus seiner Umgebung. Von ihr her werde an den Organismen eine Eigenschaft ausgewählt oder würden sie als ganze selegiert (216). Das eigene Interesse aus dem Selbstverständnis der Interpreten an der Zuchtwahl eines „Kollektivums“ statt „Individuums“ verschaffe sich hier Geltung (214).

Bleiben wir indessen bei Plessners Grenzhypothese, entsteht für das lebende Individuum ein doppeltes Selektionsproblem in einem anderen Sinne. Dieses Individuum entsteht nicht aus dem Nichts wie eine creatio ex nihilo, sondern aus allgemeinen Strukturen und Funktionen einer Art und Weise zu leben, die sich erst im Prozess entwickelt. Es individualisiert diese allgemeine Lebensart nicht nur in dem Sinne, dass die allgemeine Form der Art hier und jetzt immer erneut auf andere inhaltliche Herausforderungen durch Bedingungen im leeren Raum und in der leeren Zeit trifft. Vielmehr ist es für sich auch unumkehrbares Leben, das sich nicht als Ganzes vertreten, austauschen und ersetzen kann. Es individualisiert seine Spezies auch in dem Sinne, dass es als unteilbares sie immer erneut hier und jetzt vollzieht, nämlich in seinem Vorwegsein erfüllt. 
Das werdende Individuum gerät infolgedessen in ein doppeltes Missverhältnis zur Weite der Form, die ihm Spielraum und darin den Rahmen notwendig zu versäumender Möglichkeiten gibt, und zur Fülle seiner eigenen Positionalität, die es ihm gestattete, die gebotenen Möglichkeiten zu verwirklichen. (215)

Es schlägt zwar „überhaupt einen bestimmten Weg ein“, aber in dieser lebensnötigen Möglichkeit besteht auch „der Zufall, dass es gerade dieser und kein anderer sein musste“ (ebd.). Insoweit lässt sich kategorial sagen: „Für das Leben hat den Wert eines inneren Strukturgesetzes (man könnte es als das Gesetz des kategorischen Konjunktivs bezeichnen, das den inneren Mechanismus der Selektion ausmacht), dieses ,es ginge zwar, aber es geht nicht““ (216).

In Plessners Relativierung der biologischen Adaptations- und Selektionstheorien zugunsten eines weiteren kategorialen Lebensverständnisses entspricht doch seiner eigenen Grenzhypothese, dass Grenze nicht nur den Vollzug des Überganges bedeutet, sondern eben auch die Abschließung des physischen Körpers. Für den Ausgleich in dem „Konflikt zwischen dem Zwang zur Abgeschlossenheit als physischer Körper und dem Zwang zur Aufgeschlossenheit als Organismus“ seien zwei Weisen der Organisation, d. h. der "Selbstvermittlung der Einheit des belebten Körpers durch ihre Teile“ (185), möglich. Die offene Organisationsform gliedert den Organismus in allen seinen Lebensäußerungen unmittelbar der Umgebung ein und macht ihn zu einem unselbständigen Abschnitt seines Lebenskreises (219). Die geschlossene Organisationsform gliedert ihn in allen seinen Lebensäußerungen mittelbar seiner Umgebung ein und macht ihn zu einem selbständigen Abschnitt seines Lebenskreises (226). Im ersten Falle der offenen Organisationsform verhält sich der Organismus gleichsinnig mit seinem Positionsfeld, wozu in der Anschauung von Pflanzen passt, dass sie gepfropft und gesteckt (als wären sie ein Dividuum) werden können, sich ontogenetisch frühe Phasen im Aufbau des Individuums erhalten, die Bildung von Oberflächen eher nach außen als wie beim Tiere nach innen verläuft, die Bewegungen eher an der Pflanze als von ihr aus (wie beim Tiere) gehen. Im zweiten Falle der geschlossenen Organisationsform kann sich der Organismus auch gegensinnig zu seinem Positionsfeld verhalten, insofern er sich sowohl von seinem physischen Körper als auch seiner Umgebung abhebt. Die Gegensinnigkeit seiner Organe betrifft nicht nur deren Funktion, sondern auch Organisationsweise, weshalb der Ausgleich dieser Gegensinnigkeit zentralnervöser Ausdifferenzierung bedarf. Diese Ausdifferenzierung erfolge in einem Spektrum von mehreren Zentren für spezielle Funktionen bis zu einem Zentrum der Generalisierung von Funktionen. Die zentralnervöse Repräsentation der Organe nach innen und des sensomotorischen Funktionskreises nach außen führt in den Kontakt des Organismus zur Umwelt eine Vermittlung ein, die ihn als Ganzen 
selbständiger als in der offenen Organisationsform macht (229f.). „Die Abgehobenheit vom eigenen Leibe ermöglicht den Kontakt mit einem vom Leibe abgehobenen Sein. Der Körper ,merkt' das Sein und ,wirkt auf' das Sein. Über eine Kluft hinweg steht er mit dem Anderen in sensorischer und motorischer Verbindung“(232).

\subsection{Die Ermöglichung personalen Lebens aus der exzentrischen Positionalitätsform im Unterschied zur zentrischen Positionalitätsform}

Fragt man sich, zu welcher Positionalitätsform die zentrisch geschlossene Organisationsform passen könnte, so gerät man, solcherart Organismen folgend, in eine zentrische Positionalitätsform. Plessner nennt den Zusammenhang der „Eingespieltheit“ (202) von Organisationsform und Positionalitätsform aufeinander kurz „Sphären“ $(237,288)$ des Lebens. Die zentrisch geschlossene (im Unterschied zu der über dezentral mehrere Funktionszentren geschlossenen, 245) Organisationsform, kurz: zentrische Organisationsform ist in ihrer physischen Funktion auffällig. Einerseits bleibt ihr Zentralorgan (im entwickelten Falle das Gehirn der Säuger) als Organ vom Gesamtorganismus abhängig und in diesen eingebettet. Andererseits hebt sich dieses Organ vom Gesamtorganismus dadurch ab, dass in ihm funktional eine ,zentrale Repräsentation aller Glieder und Organe“ (231, 237) stattfindet. Der Gesamtkörper verdoppelt sich, insofern er sich zum Gesamtorganismus einschließlich des Zentralorgans organisiert, aber auch, insofern das Gesamt dieser Selbstorganisation erst durch das Zentralorgan organisiert wird, was gegensinnig und gleichsinnig zum Gesamtorganismus erfolgen kann. Dieses Lebewesen ist schon seiner Organisation nach ein Wesen vom Typ eines spontanen Subjekts: „Es ist selbst - in ihm“ (237). Dieser Organismus ist „eine Abhebung von ihm in ihm, so dass er über sich (in ihm) zu stehen kommt“ (243). Die Regulierung der Mannigfaltigkeit von Vorgängen erfolgt nach innen in der Regel auf unbewusste und nicht bewusstseinsfähige Weise, von innen werden Empfindungen bewusst. Mit dieser Entlastung nach innen korrespondiert im Verhalten nach außen und von außen eine neue, bewusstseinsfähige und auch bewusst werdende Art und Weise, sich zu positionieren. „Jedes Tier ist der Möglichkeit nach ein Zentrum, für welches (in einem wie wechselnden Umfang immer) eigener Leib und fremde Inhalte gegeben sind. Es lebt körperlich sich gegenwärtig in einem von ihm abgehobenen Umfeld oder in der Relation des Gegenüber. Insofern ist es bewusst, es merkt ihm Entgegenstehendes und reagiert aus dem Zentrum heraus, d. h. spontan, es 
handelt“ (240). Seine Positionierungsweise ist konzentrisch: „In seiner Abgehobenheit vom eigenen Leibkörper bildet es zugleich die Mitte, um welche der Körper geschlossen ist, gegen welche der Körper und das ihn umgebende Positionsfeld total konvergieren“ (237f.).

Schaut man aus dieser konzentrischen Positionierungsweise zurück, so entspricht ihr in der zentrischen Organisationsweise: Der Ringschluss des sensomotorischen Verhaltens öffnet sich: Je vermittelter das Zentralorgan, in sich zirkulär über Gedächtniskorrelate fungierend, zwischen die sensorischen und motorischen Organe tritt, desto stärker „unterbricht“, „pausiert“ (244f., 260) es deren Kopplung zum Verhaltensvollzug. Desto „gehemmter“ und verzögerter (weniger „instinktiv“, weniger „reflexhaft“ sofort) „antwortet“ (245) das Lebewesen auf Vorkommnisse in seinem Positionsfeld, aber auch desto „fehlbarer“ $(245,250)$ und „intelligenter“ im Dienste seiner aufgeschobenen, nicht aufgehobenen Trieberfüllungen. In der bewussten Verschränkung des sensorischen Merkfeldes und des motorischen Wirkfeldes entstehen ihm Dinge, an deren Signalgestalten es seine Handlungsmöglichkeiten wahrnimmt. Das Umfeld präsentiert sich nicht mehr nur in komplexen Qualitäten (wie bei dezentraler Schließung der Organisationsform, 264), sondern in Dingkonstanten, insoweit sich „Signalfeld und Aktionsfeld in Einem“ (252) überlagern.

Der Umschlag vom „Sein ins Bewusstsein“ (243) entspricht der Kippbewegung von der Vorangepasstheit (durch Instinkte, 286) in eine akute Anpassung, die aber auf der Selektivität beruht. „Die Triebrichtung ist das Selektionsprinzip des Gedächtnisses“ (285).

Die „Ablagerung dessen, was dem Lebewesen begegnet ist, in’s Gedächtnis“ erfolge „nur über seine Zukünftigkeit hin, $d$. h. vermittelt durch sein Vorwegsein“: Auf diesem „,Umweg“ (der inneren Pause)“ (organisatorisch gesagt: der zentralnervösen Filtrierung und relationalen Neukombination) werden sowohl „die Dekomposition des Erlebten in seine Elemente“ als auch deren Reund Neu-Komposition zum Erlebbaren und Vorstellbaren möglich (ebd.). „Auf jeder Stufe, bewusst oder außerbewusst, ist die historische Reaktionsbasis eine Einheit von Residuum und Antizipation oder, äquivalent gefasst, auf keiner Stufe ist die Vergangenheit, wie sie das lebendige Individuum behält, eine abgeschlossene Größe“ (286).

Plessner hat dieses Verständnis der zentrischen Lebenssphäre auch angesichts der Versuche von Wolfgang Köhler mit Schimpansen, unseren nächsten Verwandten unter den Primaten, rekonstruiert. Köhler hatte gezeigt, dass Schimpansen zweifellos eine praktische Intelligenz unter Beweis stellen können, die allerdings noch an ihren Organismus als Bezugspunkt gebunden bleibe. Dabei war unter Intelligenz eine solche Problemlösung auf dem Wege einer 
Trieberfüllung verstanden worden, die weder angeboren noch bislang erlernt sein konnte. Allgemeiner bekannt geworden sind heute nicht nur die Kombinationen von Stöcken und Kisten oder die Herstellung von Werkzeugen, um an Futter gelangen zu können, sondern auch die Fähigkeiten von Schimpansen und Bonobos zum Erwerb der Menschensprache auf dem Umweg über die Yerkes-Tastatur, wenn sie bereits unter Menschen aufwachsen. Gleichwohl hört ihr Spracherwerb vom Niveau her da auf, wo er für Menschen spezifisch wird, d. h. im dritten Lebensjahr von Menschenkindern. Schimpansen bzw. Bonobos verstehen nicht eine Sprache, die syntaktisch allgemeinen Markierungen folgt, in denen sich Narration und Diskurs von ihrer Bezeichnung der gedächtnisgestützten Vorstellung anwesender Körperleiber befreien (s. Tomasello 2003, 4.-7. chap.). Um diese Art und Weise von Sprache, die in ihrer Verwendung syntaktischer Funktionen auf andere sprachliche Symbole wieder anderer sprachlicher Symbole verweist, zu verstehen, müsste alles Mögliche, das anwesend werden kann, vor einem Kontrast stehen, der abwesend bleiben kann, sich also nicht darin auflöst, doch bald wieder (für einen Triebhaushalt) anwesend zu werden.

Genau darin, in dem Fehlen eines Sinnes für das Negative, für das Leere und die Stille, das Abwesende und Nichts im Ganzen des Raumes und der Zeit, hatte Plessner die Grenze der praktischen Intelligenz von Schimpansen formuliert (270-276). Sie können nicht sich selbst im leeren Raum und in leerer Zeit vergegenständlichen, obgleich sie mit ihrem Leib und anderen Feldverhalten im Sinne von Dingkonstanten umzugehen vermögen. Dabei kann es auch zu Überlappungen ihrer konzentrischen Kreise untereinander, in ihren sozialen „Mitverhältnissen“ (307f.), in dem Spektrum zwischen Kampf, Flucht und interleiblicher Beziehung zwischen ihnen kommen, was man heute die „Populationskultur" von Primaten nennt (s. Krüger 2010, 3. Kap.).

Empirisch-begrifflich kann man immer neue Zwischenlevels zwischen Menschen und anderen Primaten herauszubekommen versuchen, aber kategorial hilft dies nicht weiter, um der Eigenart und Einzigartigkeit von Phänomensprüngen in der lebendigen Begegnung gerecht werden zu können. Der positionale Gegenwert der zentrischen Organisationsform besteht darin, dass dieser Organismus von sich aus auch die konzentrische Mitte des Positionsfeldes bildet. Erst für uns besteht er aus Molekülen und Geweben, könnte er als Gesamtkörper ebenso an der Peripherie des Positionsfeldes stehen, ja, von einer in die andere Umwelt einer anderen Spezies wechseln z. B. zum Spracherwerb. Dieses Wissen um solche Sachverhalte (im Unterschied zu Feldverhalten zwischen Dingkomplexen in seiner Umwelt) sollte aber nicht auf ihn selbst von uns projiziert werden. Das konzentrische Lebewesen antwortet auf solche Fragen nicht, da sie aus einer anderen Lebenssphäre kommen. 
Die Schranke der tierischen Organisation liegt darin, dass dem Individuum sein selber Sein verborgen ist, weil es nicht in Beziehung zur positionalen Mitte steht, während Medium und eigener Körperleib ihm gegeben, auf die positionale Mitte, das absolute Hier-Jetzt bezogen sind. Sein Existieren im Hier-Jetzt ist nicht noch einmal bezogen, denn es ist kein Gegenpunkt mehr für eine mögliche Beziehung da. Insoweit das Tier selbst ist, geht es im Hier-Jetzt auf. Dies wird ihm nicht gegenständlich, hebt sich nicht von ihm ab, bleibt Zustand, vermitteltes Hindurch konkret-lebendigen Vollzugs. Das Tier lebt aus seiner Mitte heraus, in seine Mitte hinein, aber es lebt nicht als Mitte. [...] es bildet ein auf es selber rückbezügliches System, ein Sich, aber es erlebt nicht - sich. (288)

Wie ist es möglich, zur positionalen Mitte einer - um diese Mitte herum konzentrisch geordneten Umwelt - einen Abstand zu bekommen, und zwar nicht jeweils einem anderen, aber ebenso zentrischen Lebewesen, sondern demselben Lebewesen selbst (289)? Offenbar ist es diese Distanz zur allein zentrischen Lebenssphäre, die uns - Biologen und Philosophen, Laien unserer personalen Lebensführung - in den Antworten zentrischer Lebewesen auf unsere Fragen fehlt und die wir ihnen gegenüber auch in teilnehmenden Beobachtungen einnehmen können, so sehr uns ihr zentrischer Vollzug zum Mitmachen einlädt und uns auch beglücken kann, da er nicht unsere Verhaltensprobleme kennt. Innerhalb der zentrischen Organisationsform würde ein zweites Zentralorgan nicht weiterhelfen. Jedes Gehirn funktioniert schon in sich auch gegensinnig, heute sagt man durch Metarepräsentationen. Ein zweites Gehirn im gleichen Gesamtkörper würde das Verhalten paralysieren oder zurückführen auf die dezentrale Schließung der Organisationsform, also mehrere Zentren für verschiedene Funktionen des Verhaltens. Die Antwortrichtung muss in der Veränderung der Positionalitätsform liegen, ohne aus der bereits zentrischen Organisationsform ausbrechen zu können (291). Wie könnte die positionale Mitte auf Distanz zu sich kommen, außer sich geraten?

Die Aufgabe einer „exzentrischen Positionalität“ (292) besteht demnach darin, die Exzentrierung der positionalen Mitte zu erreichen, ohne die Positionalität der lebendigen Natur zu verlassen. Die Exzentrierung muss auch eine „volle Rückwendung“ auf das „Zentrum totaler Konvergenz des Umfeldes und des eigenen Leibes“ (ebd.) ermöglichen, also mit einer im Prinzip zentrischen Organisationsweise verträglich bleiben. Diese Aufgabe wird in der Einführung des Personbegriffes gestellt: „Positional liegt ein Dreifaches vor: das Lebendige ist Körper, im Körper (als Innenleben oder Seele) und außer dem Körper als Blickpunkt, von dem aus es beides ist. Ein Individuum, welches derart dreifach charakterisiert ist, heißt Person“ (293). Dem personalen Lebewesen bleibt der „Umschlag vom Sein innerhalb des eigenen Leibes zum Sein außerhalb des Leibes ein unaufhebbarer Doppelaspekt seiner Existenz, ein wirklicher Bruch seiner Natur“: Es „lebt diesseits und jenseits des Bruches, als Seele und als Körper und 
als die psychophysisch neutrale Einheit dieser Sphären“ (292). Aber wie ist es möglich, dass sich das personale Lebewesen von seiner positionalen Mitte im Verhalten ex-zentrieren und auf diese Mitte re-zentrieren kann? Indem der Grenzübergang, in der Exzentrierung sich zugleich zu rezentrieren, vollzogen wird. Dieser Vollzug geht nur, wenn dieses Lebewesen nicht mehr in einer konzentrischen Umwelt, sondern in einer in sich doppelt strukturierten Welt lebt. Eine solche Welt besteht im Sinne einer lebensnötigen Möglichkeit von vornherein aus Exzentrierungs- und Rezentrierungsrichtungen des Verhaltens. Alles, was und wer hier und jetzt begegnen kann, wartet gleichsam darauf, in solchen Verhaltungsperspektiven verschränkt zu werden.

Insofern die Person von ihrer konzentrischen Mitte im Positionsfeld nach außen gerichtet abrückt, rückt ihre Mitte für die Person in die Außenwelt ein. „Das von Dingen erfüllte Umfeld wird die von Gegenständen erfüllte Außenwelt, die ein Kontinuum der Leere oder der räumlich-zeitlichen Ausdehnung darstellt. Unmittelbar bezogen auf die Körpergegenstände sind die Leerformen Raum und Zeit, sofern die Gegenstände in ihren Formen Seiendes manifestieren, Manifestationsweisen des Nichts“ (293). Steht das Lebewesen außer sich, d. h. „im Nichts seiner Grenze, so steht auch das Körperding der Umwelt ,in“ der ,Leere“ relativer Örter und Zeiten“, in den reversiblen Korrelationen eines „richtungsrelativen Raum-Zeit-Ganzen“ (294). Dieses Ganze hinter dem Rahmen („Horizont") bleibt abwesend und gibt in dem Rahmen perspektivisch möglicher Richtungen nur „Fragmente“ frei (293). Aber in dieser möglichen Verkörperung von allem lässt sich hier und jetzt für den Organismus in seiner Position (294) nicht die Einheit des personalen Verhaltens vollziehen. Dafür braucht er innerhalb der Außenwelt positionalen Vollzug „,in der Mitte einer Sphäre“ (ebd.).

Der Exzentrizität der Struktur des Lebewesens entspricht die Exzentrizität der Lage oder der unaufhebbare Doppelaspekt seiner Existenz als Körper und Leib, als Ding unter Dingen an beliebigen Stellen des einen Raum-Zeitkontinuums und als um eine absolute Mitte konzentrisch geschlossenes System in einem Raum und einer Zeit von absoluten Richtungen. (Ebd.)

Exzentrisches Positionieren erfordert schon innerhalb der Außenwelt, die Sinnrichtungen von Körpern und Leibern zu verschränken.

Rückt die Person von ihrer konzentrischen Mitte im Positionsfeld nach innen gerichtet $\mathrm{ab}$, gerät sie mithin in Richtung ihres Organismus, rückt ihre Mitte für die Person in die Innenwelt ein, die den Gegensinn zur Außenwelt darstellt. Sie verhält sich nun „,im’ Leib“ in Richtung auf „den Fluchtpunkt der eigenen Innerlichkeit“ am „unobjektivierbaren Ich“, das „hinter“ Körper und Leib den Horizont bildet (295). Obgleich der Richtungssinn und die Wertigkeit von Innen- 
und Außenwelt verschieden sind, handelt es sich doch in beiden Welten um „dieselbe Struktur als die Erscheinung eines nicht ausschöpfbaren Seins, als das Gefüge aus Schale und Kern“ (ebd.), womit wir wieder am Anfang (1.4.) wären. Gerät der Person ihr leiblicher Vollzug zum Gegenstand (,in Gegenstandsstellung“), geht es um eine wahrzunehmende Wirklichkeit, in der ihre Seele disponiert erscheint und „zugleich erlebnisbedingend und erlebnisbedingt“ verstanden wird. Gerät die Person in „Selbststellung“, vollzieht sie also, sei es „naiv“ oder „reflektiert“, so „erlebt“ sie, „wird“ sie ihrer Erlebnisse „inne“ in einer „durchzumachenden Wirklichkeit“ (296). Die Selbststellung in der Innenwelt reicht in der „Skala“ vom „mir-zu-Mute Sein“ bis zum bloßen „Etwas Sein“, von der „Hingenommenheit und Selbstvergessenheit“ über die „Anmutungen“ durch Sympathie und Antipathie bis zum „,versteckt vorhandenen verdrängten Erlebnis“ (296f.). Der Primat der Selbststellung (die sog. Eigentlichkeit) über die Gegenstandsstellung verdecke die Kulturen vergleichenden Möglichkeiten schlechten und guten Schauspielens (298), in denen die „wirkliche Innenwelt" als Strukturproblem hervortrete, auf das die Kulturen verschieden antworten: „Wirkliche Innenwelt: das ist die Zerfallenheit mit sich selbst, aus der es keinen Ausweg, für die es keinen Ausgleich gibt“" (299).

Aber woher wird denn die Unterscheidung zwischen der Innen- und der Außenwelt ermöglicht? In dieser Frage, die weder in den Monismen noch Dualistismen gestellt wird, besteht Plessners entscheidende Wendung der gesamten Rekonstruktion derjenigen Präsuppositionen, die für naturwissenschaftliche Vergleiche in Anspruch genommen werden: Aber haben nicht wir selbst, d. $\mathrm{h}$. andere Personen, angeschaut, wie die erste Person in ihrer Außen- und Innenwelt auftrat? - Indem sie nämlich nach außen neben und nach innen hinter ihrer konzentrischen Mitte im Vollzug zum Vorschein kam, als spielte sie eine Rolle, in der sie sich nun verdoppeln kann? Nur die Mitwelt als das Dritte ermögliche die Unterscheidung zwischen der Außen- und Innenwelt:

Die Exzentrizität, auf welcher Außenwelt (Natur) und Innenwelt (Seele) beruhen, bestimmt, dass die individuelle Person an sich selbst individuelles und ,allgemeines' Ich unterscheiden muss. Allerdings wird ihr dies für gewöhnlich nur fassbar, wenn sie mit anderen Personen zusammen ist, und auch dann tritt dieses allgemeine Ich nie in seiner abstrakten Form, sondern mittels der ersten, zweiten, dritten Person konkret auf. Der Mensch sagt zu sich und anderen Du, Er, Wir -, nicht etwa darum, weil er erst auf Grund von Analogieschlüssen oder einfühlenden Akten in Wesen, die ihm am konformsten erscheinen, Personen annehmen müsste, sondern kraft der Struktur der eigenen Daseinsweise. (300)

Wer nach außen und nach innen außer sich, d. h. aus der positionalen Mitte heraus, gerät, steht in der Verhaltensbildung in der Aufgabe, wie er/sie/es im 
Außer-sich-Sein bei sich selbst bleiben kann. „Die Mitwelt trägt die Person, indem sie zugleich von ihr getragen und gebildet wird. Zwischen mir und mir, mir und ihm liegt die Sphäre dieser Welt des Geistes“, die „Wir-Form des eigenen Ichs" (303). Als Glied der Mitwelt stehe jede Person da, wo die andere steht (304), d. h. im Außer-sich-Sein sowohl der Innen- als auch der Außenwelt, dessen Vollzug ein mit sich selbst verlangt.

Das Rückschlussverfahren auf die Ermöglichungsstrukturen der Unterscheidung personalen Lebens von anderen Lebensformen erklärt nicht dessen Entstehung und Interpretation im Laufe der Geschichte, wohl aber das dort Erklärungsbedürftige. Für uns heute ist die Interpretation der mitweltlichen Struktur der Beziehung von dem/der Anderen auf mich und von mir auf mich zurück durch „Einzahl und Mehrzahl“ (305) in der Verwendung der Personalpronomen geläufig. Die „Individual- und Kollektiventwicklung“ zeige indessen eine „ursprüngliche Tendenz zur Anthropomorphisierung und Personifizierung“, die Folgendes verdeutliche: „Bei der Annahme der Existenz anderer Iche handelt es sich nicht um Übertragung der eigenen Daseinsweise“ auf diese Anderen, sondern um eine

[...] Einengung und Beschränkung dieses ursprünglich gerade nicht lokalisierten und seiner Lokalisierung Widerstände entgegensetzenden Seinskreises auf die ,Menschen‘. Das Verfahren der Beschränkung, wie es sich in der Deutung leibhaft erscheinender Lebenszentren abspielt, muss streng getrennt werden von der Voraussetzung, dass fremde Personen möglich sind, dass es eine personale Welt überhaupt gibt. (301)

Das Menschsein und Schimpansesein wird von der Personalität in den genannten Welten als den Präsuppositionen der künftigen Lebenswelt und Forschungspraktiken ermöglicht, nicht umgekehrt. „Daher steht es dem Menschen versuchsweise frei, diese Ort-Losigkeit der eigenen Stellung, kraft deren er Mensch ist, für sich selber und jedes andere Wesen in Anspruch zu nehmen auch da, wo ihm Lebewesen gänzlich fremder Art gegenüberstehen“ (300).

Folgt man personalen Lebewesen in ihre Positionierungsrichtungen, ergeben sich nicht nur die drei Welten als die Ermöglichungsstruktur ihres Verhaltens. Geht man in diesen Welten auf diese Lebewesen zurück, versteht man den Aufgabencharakter in ihrer Verhaltensbildung, eben den Bruch zwischen den Ex- und Re-Zentrierungen als Einheiten zu vollziehen. Erst auf diese Art und Weise kann personales Leben prozessieren und eine Entwicklungsrichtung (s. 0. 1.4.) gewinnen. Um diesen Aufgabencharakter herauszuarbeiten, spricht Plessner am Ende seiner naturphilosophischen Fundierung von drei „anthropologischen“, also die Vollzugseinheit des Bruches wahrenden „Grundgesetzen“. Es geht hier nicht um Kausalgesetzte, sondern um Gesetze, nach denen man ver- 
stehen kann, wie das Potential der exzentrischen Positionalität verwirklicht werden kann, ohne in den Bruch von Konzentrik und Exzentrik in der Verhaltensbildung auseinanderfallen zu müssen.

Beginnen wir mit der Außenwelt der Mitwelt. Wer in der Positionierung in der Außenwelt außer sich gerät, muss sich erst zu dem machen, was er/sie/es ist (310). Personalität muss den Hiatus, ihre Gestelltheit in den Bruch, als Einheit vollziehen können. Dafür müssen personale Lebewesen „,von Natur aus künstlich“ sein können: „Als exzentrisches Wesen nicht im Gleichgewicht, ortlos, zeitlos im Nichts stehend, konstitutiv heimatlos, muss er [der Mensch: HPK] ,etwas werden' und sich das Gleichgewicht - schaffen. Und er schafft es nur mit Hülfe der außernatürlichen Dinge, die aus seinem Schaffen entspringen, wenn die Ergebnisse dieses schöpferischen Machens ein eigenes Gewicht bekommen“ (310). Auf diesem Wege, die „Ergänzungsbedürftigkeit“ des personalen Lebewesens in einer Welt von Eigengewicht zu erfüllen, winkt ihm das Gleichgewicht „gleichsam in einer zweiten Natur, die Ruhelage in einer zweiten Naivität“ (311). So dient es der „Kultur“ und bedient sich aus ihr als „Werkzeug“ (ebd.). Ihm ist der Umweg über die Kultur „natürlich“, d. h. „natürliche Künstlichkeit“ (309).

Setzen wir in der Innenwelt der Mitwelt fort: Plessner nennt die Positionierungsrichtung aus der Innenwelt hinaus in ihren Vollzug „Expressivität“ und in sie hinein „Reflexivität“. Insofern die Person in der reflexiven Richtung hinter sich stehe, kann ihr einsichtig werden, dass ihr eigenes Bewusstsein den Kontakt mit der Wirklichkeit vermittelt. Alles, was sie erfährt, erfährt sie ,als Bewusstseinsinhalt und deshalb nicht als etwas im Bewusstsein, sondern außerhalb des Bewusstsein Seiendes“ (328). Trennt die Reflexion aber das vermittelnde Bewusstsein von der Unmittelbarkeit ab, die von ihm vermittelt wird, findet für diese Reflexion alles nur im Bewusstsein (seiner „Immanenz“, 329) statt. In der gegensinnig expressiven Richtung „des erfahrenden, wahrnehmenden, anschauenden, inne werdenden, verstehenden Wissens selber muss dem Menschen die Wissensbeziehung unmittelbar, direkt sein“ (328). Gleichwohl kann eine andere Person (der Mitwelt) einsehen, dass „das Wissen vom Objekt“ die Vermittlung zwischen der Person und dem Objekt darstellt. „So tilgt die Vermittlung im Vollzug ihn, den Menschen, als das hinter sich stehende vermittelnde Subjekt, es vergisst sich (er vergisst sich nicht!) - und die naive Direktheit mit der ganzen Evidenz, die Sache an sich gepackt zu haben, kommt zustande“ (ebd.). Insoweit die Unmittelbarkeit des Vollzuges nur auf dem Wege der Vermittlung gelingen kann, unterliegt die personale Verhaltensbildung einer „vermittelten Unmittelbarkeit“ (321) oder „indirekten Direktheit“ (324) Sie besteht in allen Symbolisierungsweisen des personalen Lebens, insbesondere der Sprache (340). 
Was schließlich die Mitwelt der Mitwelt angeht, so führt die Exzentrierung auch noch der Mitwelt aus ihr heraus, über sich hinaus in ihre eigene Nichtigkeit. Von dieser Ex-Position außerhalb der Mitwelt her steht die Mitwelt nur noch durch jeden „hindurch“: „Er ist die Menschheit, d. h. er als Einzelner ist absolut vertretbar und ersetzbar. Jeder andere könnte an seiner Stelle stehen, wo er mit ihm in der Ortlosigkeit exzentrischer Position zu einer Ursprungsgemeinschaft vom Charakter des Wir zusammengeschlossen ist“ (343). Dieser Nichtigkeit könne durch Religiosität begegnet werden, die die Mitwelt transzendiert und so ein „Definitivum“ schaffe: „Das, was dem Menschen Natur und Geist nicht geben können, das Letzte: so ist es -, will sie ihm geben“ (342). Gleichwohl kann dieser „Theomorphismus“ auch als nur verlängerter „Anthropomorphismus“ (345) in der exzentrischen Positionalität durchschaut werden und Zweifel wecken. Rezentriere man indessen solche Transzendenzen, komme man in den „öffentlichen Realisierungmodus der Gesellschaft“ als Mitwelt hinein (ebd.). Auf die Gefahr, in jedermann austauschbar und vertretbar, mithin nur zufällig Individuum zu sein, antworte ein zweideutiger Drang der Individualität lebender Personen, einerseits „nach Offenbarung und Geltung“ und andererseits „nach Verhaltenheit“ (344). Die Utopie der Ursprungsgemeinschaft revolutioniere die Nichtigkeit der Mitwelt und könne zum „Mittel der Erneuerung der Gesellschaft“ im Sinne der „Respektierung des Anderen“, die „Distanz und Verdecktheit gebietet“ (345), werden. Personales Leben werde durch einen „utopischen Standort“ (341) zwischen der Nichtigkeit und der Transzendenz der Welt ermöglicht. Darauf gehe ich im 3. Kapitel und im dritten Teil des vorliegenden Buchs genauer ein.

\subsection{Zur neueren Rezeption der Stufen}

Die Semantik des Lebens ist heute eine andere als vor einem ganzen oder vor einem halben Jahrhundert. Im Lebendigen schwingt noch ein Rest von Spontaneität, Freiheit und Spielerischem mit, aber nicht mehr jener Zauber, der die Kraft und „Freude an der Dämonie der unbekannten Zukunft“ (4) zum Ausdruck brächte und zum Symbol machen würde, eine ursprünglich Nietzscheanische Dämonie, die Heidegger in die Semantik der Existenzialität zu übertragen versucht hat. Das Leben selbst ist inzwischen ökonomischen, biologischen und politischen Rationalisierungsprozessen unterworfen worden, deren Produktivität im Vergleich mit früheren Jahrhunderten Michel Foucault herausgearbeitet hat, ohne allerdings eine eigene Naturphilosophie entwickelt zu haben (Krüger 2009a, 40-53). Plessner selbst sah neue Formen von „Lebensmacht“ im „Hochkapitalismus“ (4) entstehen, so exemplarisch in der Verknüpfung einer rein 
naturwissenschaftlichen statt lebensweltlichen Krankheitsdefinition mit den neu geschaffenen Absatzmärkten der Pharmaindustrie und den neuen Institutionen sozialer Hygiene (Plessner 1982e, 97-101). Gegen Heidegger bewahrheitet sich Plessners These, dass primär der geschichtliche Lebensprozess die Existenz der Einzelnen fundiert und nicht umgekehrt die je individuelle Existenz den ökologischen, ökonomischen, politischen und geistig-kulturellen Lebensprozess (XIII). Auch mit Karl Löwith gesprochen, auf den Plessner verweist: Nicht in der Teilnahme an der öffentlichen Gestaltung des geschichtlichen Lebensprozesses, auf die Plessner politisch orientiert, ist man „privativ“, sondern umgekehrt: Man beraubt sich in der Flucht in die je eigene Privatexistenz der für personales Leben nötigen Selbstverdoppelung in eine private und eine öffentliche Person (XII; vgl. Plessner 1983e, Plessner 1982i). Auch der private Rückzugsort ist längst nicht mehr sicher inmitten der Vernetzung neuer Informationsmit neuen Lebenstechnologien wie der synthetischen Biologie. Wer der ökonomischen und etatistischen Verwertung solcher synthetischen Machbarkeit keinen wirksamen demokratisch-gewaltenteiligen, rechtsstaatlichen Rahmen setzt, in dem das öffentlich-private Doppelgängertum von Personen qualitativ gesichert werden kann, verliert auch das Asyl seiner respektive ihrer Eigentlichkeit. Der Rückzug ins individuell Eigentliche lässt sich auch nicht mehr durch Selbstermächtigung der eigenen Gemeinschaftlichkeit zur Weltherrschaft, wie in Heideggers Rektoratsrede und Schwarzen Heften geschehen, kompensieren, sondern zivilisatorisch gesehen so nur noch umso destruktiver steigern.

Als charakteristisch für die neue Rezeptionslage, in die Plessners Werk während der letzten Jahrzehnte geraten ist, kann der Rückgriff des späten (im Unterschied zum mittleren) Habermas auf Plessners Körper-Leib-Differenz von Personen gelten, um in der folgenden fraglichen Lage philosophischanthropologische Orientierung finden zu können. Worin bestehen die absehbaren Folgen der marktliberalen Freigabe von Technologien der Genveränderung? Noch steht die Verhütung von Erbkrankheiten (negative Eugenik) im Vordergrund, aber es handelt sich auch schon um ein weit darüberhinausgehendes Enhancement (positive Eugenik) nach welchen Kriterien? Kann noch eine Identität der Gattung bewahrt werden, oder müssen wir uns auf alle möglichen Chimären nach neuen Klassen- und Schichtenbildungen einstellen, die mit dem gerechten Ausgleich und der Wahrung der Verhältnismäßigkeit der Grundwerte von Freiheit und Gleichheit nichts mehr zu tun haben werden (Habermas 2001)? Angesichts solcher Fragen aktualisiert Habermas Plessners Unterscheidung zwischen dem Leibsein und dem Körperhaben, in der Personen leben.

Obgleich Personen (als Glieder einer Mitwelt, 304) in einer exzentrischen Distanz von ihrer leiblichen Konzentrik stehen, fallen sie doch im lebendigen Voll- 
zug ihrer Personalität mit ihrer Leiblichkeit zusammen. Sie sind lebendig ihr Leib, den sie nur in Grenzen als Körper wie andere Körper auch haben können. Sie haben ihren Leib als Körper, insofern sie ihn instrumentieren und als Medium verwenden können, was aber in der personalen Lebensführung nicht vollständig gelingt. Lachen und Weinen als Grenzreaktionen dafür, dass die Person in Beantwortung einer Situation nicht mehr angemessen, d. h. nach üblicher Bewandtnis handeln und variabel gestalten kann, sind dafür die besten Beispiele. In ihnen antwortet der auseinanderfallende Körperleib für die situationsbezogen nicht mehr selbst beherrschte Person (vgl. Plessner 1982f, 238-243, 372384). Plessner führt diese Körper-Leib-Differenz am Ende der Stufen als zwei Verhaltensweisen der Person zu ihrem Organismus (demselben Substrat) eben als Leib (Seele) und als Körper ein (292-294), was in der körperlich-leiblichen Doppelstruktur der Außen-, Innen- und Mitwelt seiner stabilen Ermöglichung nach genauer erschlossen wird. Der leibliche Teilaspekt betrifft das in der personalen Lebensführung hier und jetzt Unvertretbare, nicht Austauschbare, nicht Ersetzbare, während sich der körperliche Teilaspekt auf das in ihr Vertretbare, Austauschbare oder Ersetzbare bezieht (Krüger 2011). Schon vor Habermas hat auch Bernhard Waldenfels in seiner responsiven Phänomenologie auf die Aktualität der Plessnerschen Körper-Leib-Differenz mit dem Fokus auf Lachen und Weinen verwiesen (Waldenfels 1994).

Plessner wird aber nicht nur von anderen Strömungen erneut rezipiert. Die Philosophische Anthropologie selber hat während des letzten Vierteljahrhunderts eine Renaissance erfahren, in deren Zentrum nun Helmuth Plessners Werk, nicht mehr das von Max Scheler oder Arnold Gehlen, steht. Bei der Philosophischen Anthropologie, wie sie Plessner in seiner Antrittsrede im Groninger Exil 1936 begründet hat (Plessner 1983d, der Schnädelbach 1983, 8. Kap. folgte), handelt es sich, über die gleichnamige Subdisziplin der Philosophie hinausgehend, um eine eigenständige philosophische Richtung, die sich von anderen Strömungen wie den Existentialismen, Marxismen, den verschiedenen Lebensphilosophien, den Neukantianismen und Neohegelianismen, der Phänomenologie, dem logischen Positivismus und Kritischen Rationalismus deutlich unterscheidet (Krüger 2001, Fischer 2008). Plessner teilt mit Scheler die Einsicht, dass die Thematisierung von Personen in natürlichen und soziokulturellen Lebensprozessen erfordert, das philosophische Verfahren gegen die dualistischen Vorentscheidungen, etwas könne entweder nur physisch oder psychisch sein, zu neutralisieren $(32,36,244,92)$. Insofern sich etwas sowohl physisch als auch psychisch in seinem Vollzug zeigt, kandidiert es der phänomenologischen Anschauung nach dafür zu leben. Aber Plessner hat die Philosophische Anthropologie auch von ihrer geistesmetaphysisch positiven Begründung durch 
Scheler befreit, ohne sie wie später Arnold Gehlen in eine empirische Philosophie aufzulösen (Krüger 2006c u. 2009a, 6. Kap.). Als Anthropologie stellt sie die Frage nach dem einheitlich erfahrbaren Zusammenhang zwischen dem Natur-, Sozial- und Kulturwesen des Menschen im geschichtlichen Prozess. Als Philosophie rekonstruiert sie die lebens- und forschungspraktischen Ermöglichungsbedingungen für solche anthropologischen Untersuchungen in einer offenen $\mathrm{Zu}$ kunft, in der die menschlichen Lebensformen nicht das einzig bekannte Beispiel für die personale Lebenssphäre bleiben müssen.

Plessner hat die Spezifik seiner Philosophischen Anthropologie dadurch markiert, dass er sie deutlich von „anthropologischen Philosophien“ unterschieden hat, die meinen, das Wesen des Menschen abschließend bestimmen zu können, statt es für künftige geschichtliche Veränderungen aufzuschließen (Plessner 1983d, 36-39; Plessner 1983i, 242-245). So definiere Ernst Cassirer das Wesen des Menschen als das animal symbolicum in einem funktionalen, nicht substantiellen Sinne (Cassirer 1990, 51, 110) oder unterstelle die Linguistic Analysis, dass sich die Natur des Menschen durch Sprache auszeichne. Darin besteht zwar ein weitgehender Konsens der verschiedensten Philosophien seit der griechischen Antike, aber warum gilt Sprachlichkeit als Wesensmerkmal des Menschen, ist es doch nicht immer nur dieselbe Sprache und allein diese, die ihn unterscheidet? Dagegen hatte Cassirer schon zu Recht die Vielfalt der Symbolformen herausgearbeitet, war dann aber nicht mehr mit dem Problem ihrer historischen Abfolge und Gleichzeitigkeit zurande gekommen. Die Sprache allein reicht nicht aus, weder empirisch noch theoretisch, schon aus Sicht der evolutionären, vertikal und horizontal vergleichenden Anthropologie, wie in der Gegenwart Michael Tomasello gegen solche anthropologischen Philosophien einwendet (vgl. zum Verhältnis dieser zur Philosophischen Anthropologie Krüger 2010, 3. Kap.). Weder die Humanontogenese noch die Humanphylogenese lasse sich aus der Sprache ableiten, weil sie ihrerseits erst in beiden Prozessen durch eine rekursive Veränderung des Entwicklungszusammenhanges zwischen Kooperations- und Kommunikationsformen fortlaufend neu ermöglicht werde (Tomasello 2014b, 5. Kap.). Man hat der Philosophischen Anthropologie Plessners immer wieder zu Unrecht die Position einer anthropologischen Philosophie unterstellt, obwohl man es besser hätte wissen können (Rölli 2015). Demgegenüber hat Plessners fruchtbare Unterscheidung zwischen Philosophischer Anthropologie und anthropologischer Philosophie, die in den Büchern von Schnädelbach (1983) und Fischer (2008) keine Rolle spielt, eine andere, weder anthropologisch-philosophische noch dekonstruktivistische Problemgeschichte seit dem 18. Jahrhundert und insbesondere einen neuen Zugang zu dem systematischen Verhältnis der Philosophien von Plessner, 
Heidegger, Cassirer und Hannah Arendt angestoßen (Krüger 2009a, II. Teil; Wunsch 2014).

Die Renaissance der Philosophischen Anthropologie hat sich nicht nur auf die soziokulturelle Problemgeschichte dieser Strömung bezogen (Fischer 2008), sondern wegen der reflektierten und synthetisierenden Stellung Plessners zwischen den verschiedenen deutschen und französischen Philosophierichtungen eine Vielfalt problemgeschichtlicher und aktuell systematischer Vergleiche erfordert, auf die ich oben nur selektiv verweisen konnte. Es steht eine freie Rekonstruktion einer ganzen diskursiven Formation europäischen Philosophierens an, das aus der heute selbstverständlich gewordenen Aufteilung sowohl der Philosophie als auch der Biowissenschaften in lauter Spezialisierungen nur schwer zugänglich geworden ist. Eine besondere Rolle spielen dabei Arbeiten, die die verschiedenen deutschen und französischen Philosophien füreinander übersetzbar werden lassen, um angesichts ihrer Grenzen systematisch neu einsetzen zu können (vgl. Plas/Raulet 2011, Raulet/Plas 2014). So hat exemplarisch Ebke die historische Epistemologie von Georges Canguilhem und Plessners Philosophische Anthropologie im Hinblick auf die dialektische Struktur des selber lebendigen Wissens vom Leben untersucht, um aus den Aporien heraus zu gelangen, von denen die zeitgenössischen Biowissenschaften ebenso heimgesucht werden wie deren philosophische Standardkritiken (Ebke 2012). Zu den bekanntesten reduktiv-naturalistischen Kategorienfehlern der letzten beiden Dekaden gehört zweifellos die Auflösung der Personalität des Lebens in ihre neurophysischen Hirnkorrelate und im Gegenzug die philosophisch idealistische Beschwörung der autonomen Subjektivität und Sprache, wodurch sich die Aktualität cartesianischer Entweder-oder-Alternativen wieder bestätigt hat (Krüger 2007; Krüger 2010, 2. Kap.).

\subsection{Zum aktuellen Interpretationsstreit über die Stufen}

Für die neue Lektüre speziell der Stufen galt es in der angesprochenen Renaissance vor allem, Plessners biophilosophische „Wende zum Objekt“ (V, 31, 72) richtig zu verstehen, die in ihrer Darstellung vom lebendigen im Unterschied zum unbelebten Körper bis zur exzentrischen Positionalität führt. Laut Fischer (2000) kam und kommt der exzentrischen Positionalität das biophilosophische Primat der Schlüsselkategorie für die ganze Strömung zu (Fischer 2008, 520f., 549). Beaufort zeigte demgegenüber parallel, dass schon die Anschauung und feststellbare Erfahrung lebendiger Körper, also der biophilosophische Anfang der Stufen, eine bestimmte Art und Weise von exzentrischer Positionalität, also das Ergebnis der Stufen am Ende, voraussetze, so in der stets mitlaufenden Re- 
ferenz auf die biologisch restringierten Erfahrungsarten und auf die sogenannt natürliche Weltsicht im common sense aller Beteiligten. Plessners „kritischphänomenologische Grundlegung einer hermeneutischen Naturphilosophie“ nahm bereits die moderne „gesellschaftliche Konstitution der Natur“ (Beaufort 2000) in Anspruch. In der Tat glaubte Plessner nicht, aus dem natürlichen Objekt, das sich im Verlaufe der Stufen zu Subjekt-Objekt-Einheiten zu entwickeln schien, am Ende die dem Menschen wesensspezifische Objekt-Subjekt-Einheit ableiten zu können, als wäre ein Autor Gott oder die Natur während der Genesis. Sein oben erwähnter Arbeitsplan setzte die lebendigen Subjekt-Objekt-Einheiten des Menschen in naturphilosophischer (vertikaler) und geschichtsphilosophischer (horizontaler) Richtung voraus, um gegen die philosophisch und erfahrungswissenschaftlich eingebürgerten Subjekt-Objekt-Trennungen diese Lebenssphären einsehen, verstehen und systematisieren zu können. Mitscherlich hat daher den dialektischen Zusammenhang zwischen der Natur- und Geschichtsphilosophie in Plessners in sich gebrochener Lebensphilosophie herausgearbeitet und dabei die beiden Seiten sogar als gleichrangig verstanden. Die Eigenart der naturphilosophischen Deduktion bestehe nicht darin, aus etwas (entweder einem Faktum oder einem Apriori) abzuleiten, als könne man es so herstellen, sondern darin, unter dem Aspekt der neuen Verbindung dieser getrennten Seiten (des Faktischen und des Ermöglichenden) anzuschauen, zu verstehen und kategorial zu systematisieren (Mitscherlich 2007).

Lindemann hat den gesellschaftlich konstitutiven Charakter der exzentrischen Positionalität in ihrer reflexiven Sozialanthropologie weiterentwickelt. Komapatienten können nicht mehr selbst ihre personale Körper-Leib-Differenz gestalten, weshalb es zu diesem Zweck einer „soziotechnischen Konstruktion von Leben und Tod in der Intensivmedizin“ (Lindemann 2002) bedarf. Angesichts solcher Grenzfälle hat sie sowohl die theoretische Frage neu aufgerollt, welche sozialtheoretischen Annahmen das Feld sozialer Phänomene begrenzen, als auch die empirische Frage, wie faktisch die Grenze zwischen sozialen Personen und anderen Entitäten gezogen wird (Lindemann 2009). Diesen grenztheoretischen Zugang hat sie in einer „mehrdimensionalen Ordnung des Sozialen“ zu verschiedenen „Weltzugängen“ fortentwickelt. In dieser Ordnung muss nicht der Kreis legitimer Akteure, d. h. der Personen, wie in der westlichen Moderne mit Menschen zusammenfallen, sondern kann als historisch kontingent begriffen werden. Auch die Natur-Kultur-Unterscheidung muss nicht als vorgegeben vorausgesetzt werden, sondern kann als nur eine mögliche Ordnung konzipiert werden (Lindemann 2014).

Man kann natürlich versuchen, wie Lindemann die exzentrische Positionalität auch als Ausgangspunkt für eine reflexive Sozialanthropologie zu verwenden, 
insofern in der Konzeption der personalen Lebenssphäre der Übergang der Naturphilosophie in die Philosophische Anthropologie ausdrücklich erfolgt. Aber zunächst folgt das die Stufen abschließende 7. Kapitel noch der vertikalen Untersuchungsrichtung. Es expliziert diejenigen Strukturen, die es der modernen „Verstandeskultur“ (301) und der modernen Biologie ermöglichen, in vertikaler Richtung Unterscheidungen auf der Grundlage von Gemeinsamkeiten allen Lebens zu treffen. Im vorangegangenen 6. Kapitel der Stufen ging es um das Thema der „Sphäre des Tieres“ (237-287). Um die dort gewonnenen kategorialen Unterscheidungen zwischen dem dezentralen (niedriger entwickelte Tiere) und zentralen Typ (höher entwickelte Tiere) im Rahmen der geschlossenen Organisationsform und der zentrischen Positionalitätsform, die anhand von Wolfgang Köhlers Schimpansen-Experimenten untersucht wurde, zu ermöglichen, muss eine Distanz von der zentrischen Organisationsform und von der zentrischen Positionalitätsform in Anspruch genommen werden. Ohne diesen lebens- und forschungspraktischen Abstand könnten diese Organisations- und Positionalitätsformen nicht als solche im Rahmen von Gemeinsamkeiten unterschieden werden. Das Abschlusskapitel behandelt die Frage, wie es möglich war, dass die bisherigen Grenzrealiserungen lebendiger Körper angeschaut, verstanden und begriffen werden konnten. Es expliziert diejenigen modernen lebens- und forschungspraktischen Präsuppositionen, die von Anfang an in der naturphilosophischen Wende zum Objekt immer schon in Anspruch genommen worden sind. Das Abschlusskapitel holt damit die im Arbeitsplan vorausgesetzte Subjekt-Objekt-Einheit des Menschen in der Natur (vertikal verstanden) ein. Dieses Kapitel kann also nicht allein dasjenige Explikationsbedürfnis der ebenfalls im Arbeitsplan vorausgesetzten Subjekt-Objekt-Einheit des Menschen in der Soziokultur (statisch) und deren Geschichtlichkeit (dynamisch) im horizontalen Sinne befriedigen, womit sich Plessner in anderen, oben genannten Schriften beschäftigt hat. Lindemann hat daher stets ihre Stufen-Lektüre mit der von Plessners Politscher Anthropologie in seiner Schrift Macht und menschliche Natur kombiniert.

Plessner unterstellt in seinem immanent anhebenden Ansatz das in der westlichen Moderne ausgebildete Selbstverständnis von Menschen als Menschen, daher die durchgängige Redeweise ad hominem, die Adressierung seines Publikums als Menschen, nicht als Art-, Volks- oder Klassengenossen. „Dass wir Menschen sind und sein sollen, diese Entdeckung oder diese Forderung verdanken wir einer bestimmten Geschichte, der griechischen Antike und der jüdisch-christlichen Religiosität“" (Plessner 1983d, 37). Weil wir inzwischen aus historischer Erfahrung, 
[...] durch die Kritik der Entwicklungsidee, durch die politische und ideologische Bekämpfbarkeit der Humanitas um die Gewagtheit und Rückhaltlosigkeit des ,Menschen“Gedankens wissen, müssen wir das Menschsein in der denkbar größten Fülle an Möglichkeiten, in seiner unbeherrschbaren Vieldeutigkeit und realen Gefährdetheit so zum Ansatz bringen, dass die Gewagtheit eines derartigen Begriffs als Übernahme einer besonderen Verantwortung vor der Geschichte verständlich wird. (Ebd.)

Dass man sein Publikum bei dem ihm eigenen Selbstverständnis abholt, das heute als noch umstrittener als zu Plessners Zeit gelten darf, bedeutet natürlich nicht, dass man in diesem hermeneutischen Zirkel einfach verbleibt. Der oben genannte theoretisch-methodische Aufwand an Exzentrierung in dem Untersuchungsverfahren Plessners befreit die Philosophische Anthropologie vom anthropologischen Zirkel (Krüger 2001, 1. Kap.): Nicht die Redeweise „des Menschen“, sondern die „Lebenssphäre“ (288) von „Personen“ in der „exzentrischen Positionalität“ (292f.) sind die Kategorien, in denen die naturphilosophische Rekonstruktion der Ermöglichungsstrukturen endet. Man kann sich in dieser Sphäre auch anders denn als Mensch verstehen. Ob dies besser oder schlechter wäre, ist vor allem eine Frage der geschichtsphilosophischen Fundierung in horizontaler Richtung (Krüger 2013a, im vorliegenden Band III. Teil).

Es bleibt die Frage nach dem Verhältnis zwischen der natur- und der geschichtsphilosophischen Fundierung in Plessners Philosophischer Anthropologie, zwei Fundierungsrichtungen, die sich gegenseitig ergänzen und korrigieren können. Mitscherlich hat gezeigt, inwiefern sie sich gegenseitig ermöglichen, begrenzen und verschränkt sind. Gleichwohl halte ich nicht beide Untersuchungsrichtungen bei Plessner für gleichrangig. Die Naturphilosophie entdeckt den Hiatus, den Bruch mit der leiblichen Einheit des Organismus und seiner Umwelt, das Nichts und seine Manifestationsweisen, in die personale Lebenssphären gestellt sind, bevor (im logischen Sinne) Personen sich dazu stellen können (292-294). Es ist nicht selbstverständlich, dass die Seiten (körperliche versus leibliche) des Bruches in einem Dritten (Personalität, Mitwelt) verschränkt werden können, mithin dass in der Bodenlosigkeit dieses Bruches Boden zum Stehen im Leben gewonnen werden kann. Dafür müssen die drei Aufgaben, die in den anthropologischen Grundgesetzen formuliert werden, gelöst werden können (,natürliche Künstlichkeit“, 309; „,vermittelte Unmittelbarkeit“, 321; „utopischer Standort“, 341). Bekanntlich sind alle anderen als unsere Homo-Spezies ausgestorben. Auch von den ursprünglichen Zivilisationen sind nur wenige übrig geblieben. Und warum sollten wir ewig leben und die einzigen Exemplare der personalen Lebenssphäre bleiben (Plessner 1983i, 245f.)? Für Plessner hat m. E. die Naturphilosophie den Primat, weil der Hiatus zwischen Konzentrik und Exzentrik in der personalen Verhaltensbildung in eine 
Fraglichkeit von Verhaltensmöglichkeiten führt, welche nicht übergeschichtlich durch eine endgültige Soziokultur beantwortet werden kann, sondern geschichtlich für neue soziokulturelle Antworten offen bleibt. Die Fraglichkeit überschießt die Antwortlichkeit (Krüger 2006a-c), weshalb letztere erstere nicht abschließen kann. Die Fraglichkeit enthält zwar, soll es überhaupt zu einer Antwortmöglichkeit kommen, eine zu wendende Not, nämlich die zur Exzentrierung zentrischer Positionalität, aber womöglich enthält die „Natur selber“ (Löwith 1957, 85) eine Exzentrierungsmöglichkeit anderer Art, als sie uns irdisch und bislang vertraut ist (Krüger 2009a, 80-84). Was wir als Geschichtlichkeit kennen, enthält eine mythische, religiöse oder ideologische Form von Utopie, den Hiatus wirklich zu heilen, ohne dieses Versprechen vollständig und damit ein für alle Mal und für alle realisieren zu können. Es wirkt auf irreale Weise und kann sich, wird dies praktisch nicht beachtet, in seiner Realisierung verkehren, heute womöglich unter dem Banner des Post-Humanismus. Das naturphilosophische Primat des Hiatus bedeutet also nicht das Ende, sondern umgekehrt die offene Fraglichkeit personalen Lebens in seinen geschichtlichen Versuchen, auf sie zu antworten (Krüger 1998b). John McDowell hat mit diesem weiten, geschichtlich offenen Naturverständnis (im Unterschied zu naturwissenschaftlichen Erklärungen aus Naturgesetzen) sympathisiert, wenngleich sein therapeutisches Anliegen weniger ambitioniert sei (McDowell 1998, 121-125).

Mit diesen kurzen Hinweisen auf eine interessante Forschungslage dürfen wir auch in Zukunft systematisch spannende Beiträge zur Diskussion der Stufen erwarten (zum derzeitigen Stand Krüger 2017d). Zudem ist glücklicher Weise auch ein Band erschienen, der speziell den zeithistorischen Kontext der Stufen rekonstruiert hat, in dem Plessners Dialoge sowohl mit Naturwissenschaftlern als auch Philosophen stattgefunden haben (Köchy/Michelini 2015). Die systematische Aktualität der Stufen spricht Charles Taylor in seinem Verweis auf die exzentrische Positionalität an, in der man die historische Evolution der Flexibilität statt Instinkthaftigkeit des menschlichen Lebens verstehen könne (Taylor 2016, 341f.). Die angloamerikanische Diskussion von Plessners Philosophie, insbesondere seiner Naturphilosophie hat begonnen (de Mul 2014; Honenberger 2016). Auch Habermas hat jüngst erneut in einem Interview die Bedeutung von Plessners Stufen unterstrichen:

Sie haben völlig Recht, ich hätte von Plessners anthropologischen Grundeinsichten, die mir seit meinem Studium vertraut sind, explizit Gebrauch machen können - und sollen. Ich halte Die Stufen des Organischen und der Mensch neben Sein und Zeit und Geschichte und Klassenbewusstsein für das bedeutendste Werk der 20er Jahre, jenes philosophisch fruchtbarsten Jahrzehnts des 20. Jahrhunderts. (Habermas 2016, 812) 